\title{
A-infinity algebras, modules and functor categories
}

\author{
Bernhard Keller
}

\begin{abstract}
In this survey, we first present basic facts on A-infinity algebras and modules including their use in describing triangulated categories. Then we describe the Quillen model approach to A-infinity structures following K. Lefèvre's thesis. Finally, starting from an idea of V. Lyubashenko's, we give a conceptual construction of A-infinity functor categories using a suitable closed monoidal category of cocategories. In particular, this yields a natural construction of the bialgebra structure on the bar construction of the Hochschild complex of an associative algebra.
\end{abstract}

\section{Introduction}

A-infinity spaces and A-infinity algebras were invented at the beginning of the sixties by Stasheff [38. In the seventies and the eighties, they were developped further by Smirnov [37, Kadeishvili [18, Prouté [32, Huebschmann [15, 16], ... especially with a view towards applications in topology. At the beginning of the nineties, the relevance of A-infinity structures in geometry and physics became apparent through the work of Getzler-Jones [12, Stasheff [39, Fukaya [9], Kontsevich 21, ..., and later Kontsevich-Soibelman [24, Seidel [36], ... We refer to 19] 20 for a more detailed introduction with numerous references. The present survey consists of three parts:

1. In sections $1-3$, we will define $A_{\infty}$-algebras and examine their basic properties. Then we will define $A_{\infty}$-modules, the derived category and conclude with the description of triangulated categories via $A_{\infty}$-algebras.

2. In section 4 , we will present the interpretation of $A_{\infty}$-algebras (resp. $A_{\infty^{-}}$ modules) as the fibrant objects in the model category of certain differential graded coalgebras (resp. comodules) following Lefèvre [25].

3. In section 5, starting from an idea of V. Lyubashenko's [27 we will give a conceptual construction of $A_{\infty}$-functor categories and use it to construct the canonical bialgebra structure on the cobar construction of the Hochschild complex of an associative algebra.

1991 Mathematics Subject Classification. 18E30, 16D90, 18G40, 18G10, 55U35.

Key words and phrases. A-infinity structure, strong homotopy algebra, Quillen model category, triangulated category.

(c) 0000 (copyright holder) 
Acknowledgments. The first two parts of this survey are based on lectures given by the author at the Workshop on Derived Categories, Quivers and Strings (Edinburgh, August 2004) and at the Conference on Topology (Canberra, July 2003). The author is grateful to the organizers of both events and in particular to Alastair King and Amnon Neeman for their interest and encouragment. He thanks Vladimir Lyubashenko for stimulating discussions on the third part and Pedro Nicolás for carefully reading the manuscript and pointing out an error in a previous version of section 5.1 He is grateful to the organizers of the ICRA XIII for including this material in the proceedings of the workshop.

\section{A-infinity algebras}

2.1. Notations. We will follow Fukaya's sign and degree conventions. For this, we need to introduce some notation:

Let $k$ be a field. If $V$ is a graded vector space, $i . e$.

$$
V=\bigoplus_{p \in \mathbf{Z}} V^{p}
$$

we denote by $S V$ or $V[1]$ the graded space with $(S V)^{p}=V^{p+1}$ for all $p \in \mathbf{Z}$. We call $S V$ the suspension or the shift of $V$.

If $f: V \rightarrow V^{\prime}$ and $g: W \rightarrow W^{\prime}$ are homogeneous maps between graded spaces, their tensor product

$$
f \otimes g: V \otimes W \rightarrow V^{\prime} \otimes W^{\prime}
$$

is defined by

$$
(f \otimes g)(v \otimes w)=(-1)^{|g||v|} f(v) \otimes g(w)
$$

for all homogeneous elements $v \in V$ and $w \in W$.

If $V$ and $V^{\prime}$ are complexes, $i$. e. endowed with differentials $d$ homogeneous of degree 1 and square 0 , we put $d_{S V}=-d_{V}$ and, for a homogeneous map $f: V \rightarrow V^{\prime}$,

$$
d(f)=d_{V^{\prime}} \circ f-(-1)^{|f|} f \circ d_{V} .
$$

Thus, $f$ is a morphism of complexes iff $d(f)=0$ and two morphisms of complexes $f$ and $f^{\prime}$ are homotopic iff there is a morphism of graded spaces $h$ such that $f^{\prime}=$ $f+d(h)$. We will use that homotopic morphisms induce the same map in homology.

2.2. A-infinity algebras. An $A_{\infty}$-algebra is a graded space $A$ endowed with maps

$$
b_{n}:(S A)^{\otimes n} \rightarrow S A
$$

defined for $n \geq 1$, homogeneous of degree 1 and such that, for all $n \geq 1$, we have

$$
\sum_{i+j+l=n} b_{i+1+l} \circ\left(\mathbf{1}^{\otimes i} \otimes b_{j} \otimes \mathbf{1}^{\otimes l}\right)=0
$$

as maps from $(S A)^{\otimes p}$ to $S A$. Here, the symbol 1 denotes the identity map of $S A$. We visualize $b_{n}$ either as a planar tree with $n$ leaves and one root or as a halfdisk whose upper arc is divided into segments, each of which symbolizes an 'input', and whose base segment symbolizes the 'output' of the operation. 
Using this last representation, the defining identity (2.1) is depicted as follows:

$$
\sum \pm \quad=0
$$

Let us analyze the defining identity for small values of $n$ : For $n=1$, it states that $b_{1}^{2}=0$, so that $\left(S A, b_{1}\right)$ is a complex. We also make $A$ into a complex by endowing it with the shifted differential

$$
m_{1}=-b_{1} .
$$

For $n=2$, the defining identity becomes

$$
b_{1} b_{2}+b_{2}\left(b_{1} \otimes \mathbf{1}+\mathbf{1} \otimes b_{1}\right)=0 .
$$

Note that $b_{1} \otimes \mathbf{1}+\mathbf{1} \otimes b_{1}$ is the differential of $S A \otimes S A$ so that we obtain $d\left(b_{2}\right)=0$, which means that $b_{2}: S A \otimes S A \rightarrow S A$ is a morphism of complexes.

For $n=3$, the identity (2.1) becomes

$$
b_{2}\left(b_{2} \otimes \mathbf{1}+\mathbf{1} \otimes b_{2}\right)+b_{1} b_{3}+b_{3}\left(b_{1} \otimes \mathbf{1} \otimes \mathbf{1}+\mathbf{1} \otimes b_{1} \otimes \mathbf{1}+\mathbf{1} \otimes \mathbf{1} \otimes b_{1}\right)=0 .
$$

Here the second summand is $d\left(b_{3}\right)$ whereas the first is, up to a sign, the associator for the binary operation $b_{2}$. If we define $m_{2}: A \otimes A \rightarrow A$ by

$$
m_{2}(x, y)=(-1)^{|x|} b_{2}(x, y),
$$

then we obtain that $m_{2}$ is associative up to a homotopy given by $b_{3}$.

For each $n>3$, the identity (2.1) states that the maps $b_{2}, \ldots, b_{n-1}$ satisfy a certain quadratic identity up to a homotopy given by $b_{n}$. In this sense, an $A_{\infty}$ algebra is an algebra associative up to a given system of higher homotopies.

It is a direct consequence of the definition, that if $b_{n}$ vanishes for each $n \geq 3$, then $\left(A, m_{1}, m_{2}\right)$ is a differential graded $(=\mathrm{dg})$ algebra, i. e. $m_{2}$ is associative and $m_{1}$ a differential compatible with $m_{2}$ through the (graded) Leibniz rule. Conversely, each dg algebra gives rise to an $A_{\infty}$-algebra with vanishing $b_{n}, n \geq 3$.

In particular, each ordinary associative algebra can be viewed as an $A_{\infty}$-algebra concentrated in degree 0 , and conversely. 
2.3. Examples via deformations. Following an idea of Penkava-Schwarz 31, let us exhibit a large class of easily constructed but non trivial examples of $A_{\infty}$-algebras. Let $B$ be an ordinary algebra and $N \geq 1$ an integer. Let $\varepsilon$ be an indeterminate of degree $2-N$. We first endow the graded space $A=B[\varepsilon] /\left(\varepsilon^{2}\right)$ with the trivial $A_{\infty}$-structure given by the map $b_{2}$ induced by the multiplication of $B$ and the maps $b_{n}=0$ for all $n \neq 2$. Now let

$$
c: B^{\otimes N} \rightarrow B
$$

be any linear map. Define deformed multiplications

$$
b_{n}^{\prime}= \begin{cases}b_{n} & n \neq N \\ b_{N}+\varepsilon c & n=N .\end{cases}
$$

Then it is easy to see that $A$ endowed with the $b_{n}^{\prime}$ is an $A_{\infty}$-algebra iff $c$ is a Hochschild cocycle for $B$.

2.4. Weak A-infinity algebras. A weak $A_{\infty}$-algebra is a graded space $A$ endowed with maps $b_{0}: k \rightarrow S A$ and $b_{n}, n \geq 0$, such that the identity 2.1] holds for all $n \geq 0$. The preceding example then naturally extends to the case where $N=0$, where we start from a Hochschild 0-cocycle, $i$. e. a central element $c$ of $B$. In general, in a weak $A_{\infty}$-algebra, we have

$$
b_{1}^{2}=-b_{2}\left(b_{0} \otimes \mathbf{1}+\mathbf{1} \otimes b_{0}\right) \neq 0
$$

so that the homology with respect to $b_{1}$ is no longer defined and the above remarks no longer apply. Little is known about weak $A_{\infty}$-algebras in general, but they do appear in nature as deformations.

2.5. Morphisms and quasi-isomorphisms. A morphism of $A_{\infty}$-algebras $f: A \rightarrow B$ is given by maps

$$
f_{n}:(S A)^{\otimes n} \rightarrow S B, n \geq 1,
$$

homogeneous of degree 0 such that, for all $n \geq 1$, we have

$$
\sum_{i+j+l=n} f_{i+1+l} \circ\left(\mathbf{1}^{\otimes i} \otimes b_{j} \otimes \mathbf{1}^{\otimes l}\right)=\sum_{i_{1}+\cdots i_{s}=n} b_{s} \circ\left(f_{i_{1}} \otimes \cdots \otimes f_{i_{s}}\right) .
$$

By looking at this equation for $n=1$ and $n=2$ we see that $f_{1}$ then induces a morphism of complexes from $\left(A, m_{1}\right)$ to $\left(B, m_{1}\right)$ which is compatible with $m_{2}$ up to an homotopy given by $f_{2}$. In particular, $f_{1}$ induces an algebra morphism

$$
H^{*} A \rightarrow H^{*} B \text {. }
$$

By definition, $f$ is an $A_{\infty}$-quasi-isomorphism if $f_{1}$ is a quasi-isomorphism (i.e. induces an isomorphism in homology).

The composition $f \circ g$ of two morphisms is given by

$$
(f \circ g)_{n}=\sum_{i_{1}+\cdots+i_{s}=n} f_{s} \circ\left(g_{i_{1}} \otimes \cdots g_{i_{s}}\right) .
$$

The identical morphism of $S A$ is given by $f_{1}=\mathbf{1}$ and $f_{n}=0$ for all $n \geq 2$.

It is easy to see that we do obtain a category. It contains the category of dg algebras and their morphisms as a non-full subcategory.

Proposition 2.1. ( $c f$. section 4.8) For each $A_{\infty}$-algebra $A$, there is a universal $A_{\infty}$-algebra morphism $\varphi: A \rightarrow U(A)$ to a $\operatorname{dg}$ algebra $U(A)$. Moreover, $\varphi$ is an $A_{\infty}$-quasi-isomorphism. 
The universal property means that for each dg algebra $B$, each $A_{\infty}$-morphism $f: A \rightarrow B$ factors as $f=g \circ \varphi$ for a unique morphism of dg algebras $g: U(A) \rightarrow B$. The proposition tells us that, up to $A_{\infty}$-quasi-isomorphism, $A_{\infty}$-algebras are quite similar to dg algebras. However, in other respects, they are radically different from $\mathrm{dg}$ algebras, as the following proposition shows.

Proposition 2.2. Let $A$ be an $A_{\infty}$-algebra, $V$ a complex and $f_{1}: A \rightarrow V$ a quasi-isomorphism of complexes. Then $V$ admits a structure of $A_{\infty}$-algebra such that $f_{1}$ extends to an $A_{\infty}$-quasi-isomorphism $f: A \rightarrow V$.

The analogous statement for dg algebras and their morphisms is of course completely wrong. For our complex $V$, we can take in particular the graded space $H^{*} V$ with the zero differential (since we work over a field, the canonical surjection from the cycles to the homology of $V$ splits and we obtain $f_{1}$ by composing a right inverse with the inclusion of the cycles). Then we obtain the first part of the

Theorem 2.3. If $A$ is an $A_{\infty}$-algebra, then $H^{*} A$ admits an $A_{\infty}$-algebra structure such that

(1) $b_{1}=0$ and $b_{2}$ is induced from $b_{2}^{A}$, and

(2) there is an $A_{\infty}$-quasi-isomorphism $A \rightarrow H^{*} A$ inducing the identity in homology.

Moreover, this structure is unique up to (non unique) $A_{\infty}$-isomorphism.

Note that uniqueness up to $A_{\infty}$-quasi-isomorphism is trivial. The point is that here we can omit 'quasi'. An $A_{\infty}$-algebra is minimal if $b_{1}=0$. The minimal model of an $A_{\infty}$-algebra $A$ is the space $H^{*} A$ endowed with 'the' structure provided by the theorem. This structure can be computed as follows [23: Choose

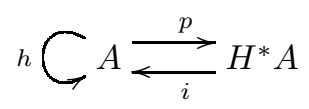

such that $p$ and $i$ are morphisms of complexes of degree 0 and $h$ is a homogeneous map of degree -1 such that

$$
p i=\mathbf{1}, i p=\mathbf{1}+d(h), h^{2}=0 .
$$

Then the $n$th multiplication of the minimal model is constructed as

$$
b_{n}^{\text {min }}=\sum_{T} b_{n}^{T}
$$

where $T$ ranges over the planar rooted trees $T$ with $n$ leaves and $b_{n}^{T}$ is given by composing the tree-shaped diagram obtained by labelling each leaf by $i$, each branch point with $m$ branches by $b_{m}$, each internal edge by $h$ and the root by $p$. 
2.6. Yoneda algebras. Let $B$ be a unital associative algebra, $M$ a (right) $B$-module and $P \rightarrow M$ a projective resolution. Let $A=\operatorname{Hom}_{B}(P, P)$ be the differential graded endomorphism algebra of $P$ (its $n$th component consists of the morphisms of graded objects of degree $n$ and its differential is the supercommutator with the differential of $P$ ). Then $A$ is in particular an $A_{\infty}$-algebra and thus has a minimal model. Now the homology $H^{*} A$ is isomorphic, as an algebra for $m_{2}$, to the Yoneda algebra

$$
\operatorname{Ext}_{B}^{*}(M, M)
$$

Thus we obtain higher multiplications on the Yoneda algebra. The simplest case where these are non-trivial is that of the algebra $B$ given by the quiver

$$
1 \stackrel{\gamma}{\longrightarrow} 2 \stackrel{\beta}{\longrightarrow} 3 \stackrel{\alpha}{\longrightarrow} 4
$$

with the relation $\alpha \beta \gamma=0$ and the module $M$ equal to the sum of the four simple $B$-modules. Then the Yoneda algebra is given by the quiver

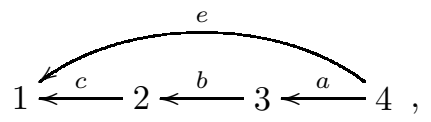

where the arrows $a, b, c$ are of degree 1 , the arrow $e$ is of degree 2 , we have $m_{2}(c, b)=$ $0, m_{2}(b, a)=0$ and $b_{3}(c, b, a)=e$.

Another example is given by the algebras $B=k[x] /\left(x^{n}\right)$, where $n$ is an integer $\geq 3$, and the simple $B$-module $M=k$. In this case, the underlying graded algebra of the Yoneda algebra is isomorphic to $k[u, v] /\left(v^{2}\right)$, where $u$ is of degree 2 and $v$ of degree 1. Note that this is independent of $n$. In contrast, the $A_{\infty}$-structure depends on $n$ : it is given by the operations $b_{l}$ which vanish for $l \neq 2, n$ such that $b_{2}$ is given by the Yoneda multiplication $m_{2}$ and $b_{n}$ is the unique $k[u]$-multilinear map which vanishes if one of the arguments is in $k[u]$ and satisfies

$$
b_{n}(v, \ldots, v)=u \text {. }
$$

This example was treated by D. Madsen 28. More generally, J. Chuang and A. King have computed the $A_{\infty}$-structure on $\operatorname{Ext}_{B}^{*}(M, M)$ for the sum $M$ of the simple modules over an arbitrary monomial algebra $B, c f$. [6]. Other examples have been computed by Lu-Palmieri-Wu-Zhang [26].

2.7. On the meaning of the higher multiplications. This section should be skipped on a first reading. Suppose that $A$ is a minimal $A_{\infty}$-algebra. Endow $H^{*}(A)$ with the associative product obtained from $b_{2}$. Then $b_{3}$ yields a 3 -cocycle homogeneous of degree -1 for the natural grading on $H^{*} A$ and thus an element in Hochschild cohomology

$$
\gamma \in H H^{3,-1}\left(H^{*} A, H^{*} A\right) .
$$

This class determines the Massey products in $H^{*}(A), c f$. for example 29. It also controls the realizability (up to direct factors) of graded $H^{*} A$-modules $M$ as the homology $H^{*} \widetilde{M}$ of an $A$-module $\widetilde{M}, c f$. $[\underline{3}$.

One can also interpret the 'higher' operations $b_{n}$. To simplify the exposition, let us change the setting slightly: Let $\mathcal{B}$ be a small $k$-linear abelian category and consider its bounded derived category $\mathrm{D}^{b}(\mathcal{B})$. Thanks to the shift functor, it becomes a graded category with morphism spaces

$$
\operatorname{Hom}^{\bullet}(L, M)=\bigoplus_{n \in \mathbf{Z}} \operatorname{Hom}_{\mathrm{D}^{b}(\mathcal{B})}(L, M[n]) .
$$


This category can be completed into a minimal $A_{\infty}$-category in much the same way that a Yoneda algebra can be completed into a minimal $A_{\infty}$-algebra. In particular, the operation $b_{3}$ determines an element in the Hochschild cohomology

$$
\gamma \in H H^{3,-1}\left(\mathrm{D}^{b}(\mathcal{B}), \mathrm{D}^{b}(\mathcal{B})\right)
$$

of the graded category $\mathrm{D}^{b}(\mathcal{B})$ (defined as in $[\mathbf{3 0}$ ). One can show that this element determines the triangulated structure of the $k$-linear category $\mathrm{D}^{b}(\mathcal{B})$. But in fact, it determines more, namely it allows one to reconstruct the $k$-linear category

$$
\mathrm{D}^{b}(\operatorname{fun}(\cdot \rightarrow \cdot, \mathcal{B}))
$$

from $\mathrm{D}^{b}(\mathcal{B})$, where $\rightarrow \cdot$ denotes the $k$-category with 2 objects and one non trivial morphism and fun the functor category. For this, one notes that $\gamma$ corresponds to $\mathrm{D}^{b}(\operatorname{fun}(\cdot \rightarrow \cdot, \mathcal{B}))$ considered as an extension of $\operatorname{fun}\left(\cdot \rightarrow \cdot, \mathrm{D}^{b}(\mathcal{B})\right)$ by the bimodule (in the sense of [30])

$$
(L \rightarrow M) \mapsto \operatorname{Hom}_{\mathrm{D}^{b}(\mathcal{B})}(L, M[1]) .
$$

In a similar manner, for $n \geq 1$, the operations $b_{2}, \ldots, b_{n+2}$ allow one to reconstruct

$$
\mathrm{D}^{b}\left(\operatorname{fun}\left((\cdot \rightarrow \cdot)^{n}, \mathcal{B}\right)\right)
$$

from $\mathrm{D}^{b}(\mathcal{B})$.

2.8. Units. A strict unit for an $A_{\infty}$-algebra $A$ is an element $1 \in A^{0}$ which is a unit for $m_{2}$ and such that, for $n \neq 2$, the map $b_{n}$ takes the value 0 as soon as one of its arguments equals 1 . Unfortunately, strict unitality is not preserved by $A_{\infty}$-quasiisomorphisms. A homological unit for $A$ is a unit for the associative algebra $H^{*} A$ with the multiplication induced by $m_{2}$. Homological unitality is clearly preserved under $A_{\infty}$-quasi-isomorphism but is not easy to handle in practical computations. Fortunately, it turns out that the two notions are not very different:

Proposition 2.4 (25, 3.2.1]). Each (resp. minimal) homologically unital $A_{\infty}$-algebra is $A_{\infty}$-quasi-isomorphic (resp. $A_{\infty}$-isomorphic) to a strictly unital $A_{\infty}$-algebra.

\section{A-infinity modules}

Let $A$ be a homologically unital $A_{\infty}$-algebra. An $A_{\infty}$-module is a graded space $M$ with maps

$$
b_{n}: S M \otimes(S A)^{\otimes n-1} \rightarrow S M, n \geq 1,
$$

homogeneous of degree 1 such that the identity 2.1 holds for all $n \geq 1$ (where we have to interpret $b_{n}$ as $b_{n}^{A}$ or $b_{n}^{M}$ according to the type of its arguments) and that the induced action

$$
H^{*} M \otimes H^{*} A \rightarrow H^{*} M
$$

is unital. For example, the $A_{\infty}$-algebra $A$ can be viewed as a module over itself: the free module of rank one. The notions of morphism and quasi-isomorphism of $A_{\infty}$-modules are defined in the natural way. The derived category $\mathrm{D}_{\infty} A$ is defined as the localization of the category of $A_{\infty}$-modules (with degree 0 morphisms) with respect to the class of quasi-isomorphisms. Thus, its objects are all $A_{\infty}$-modules and its morphisms are obtained from morphisms of $A_{\infty}$-modules by formally inverting all quasi-isomorphisms. It turns out that the derived category is naturally a triangulated category. The perfect derived category per $A$ is defined as the closure 
of the free $A$-module of rank one under shifts in both directions, extensions and passage to direct factors.

When $A$ is an ordinary unital associative algebra, we have a natural functor from the category of complexes over the category $\operatorname{Mod} A$ of (right) $A$-modules to the category of $A_{\infty}$-modules. This functor is faithful but neither full nor essentially surjective. Nevertheless, it induces an equivalence

$$
\mathrm{D}(\operatorname{Mod} A) \rightarrow \mathrm{D}_{\infty} A .
$$

Under this equivalence, the perfect derived category corresponds to the full subcategory of complexes quasi-isomorphic to bounded complexes of finitely generated projective $A$-modules.

Let $B$ be another homologically unital $A_{\infty}$-algebra. An $A_{\infty}$-bimodule is given by a graded space $X$ with maps

$$
b_{i, j}:(S A)^{\otimes i} \otimes S X \otimes(S B)^{\otimes j} \rightarrow S X, i+j \geq 0,
$$

satisfying the identity 2.1 for all $i, j \geq 0$ and such that $H^{*} X$ becomes a unital $H^{*} A-H^{*} B$-bimodule. For such a bimodule, one can define the tensor product

$$
? \stackrel{\infty}{\otimes}{ }_{A} X: \mathrm{D}_{\infty} A \rightarrow \mathrm{D}_{\infty} B, M \mapsto \bigoplus_{i=0}^{\infty} M \otimes(S A)^{\otimes i} \otimes X
$$

(we only indicate the underlying graded space). The $A_{\infty}$-algebras $A$ and $B$ are derived equivalent if there is an $A_{\infty}$-bimodule $X$ such that the associated tensor product is an equivalence.

This generalizes the now classical notion of derived equivalence for ordinary algebras: If $A$ and $B$ are ordinary algebras, then, by J. Rickard's theorem [34, they are derived equivalent iff $B$ admits a tilting complex (e.g. a tilting module) with endomorphism ring $A$.

Let us call algebraic a triangulated category which is the homotopy category associated with a stable $k$-linear Quillen model category [14] (recall that $k$ is the ground field). The class of algebraic triangulated categories contains all homotopy categories of complexes over $k$-linear categories and is stable under passage to triangulated subcategories and Verdier localizations. Thus, it contains all triangulated categories 'of algebraic origin', e. g. derived categories of categories of coherent sheaves.

Recall that an additive category has split idempotents if every idempotent endomorphism admits a kernel (and thus gives rise to a direct sum decomposition). By a generator of a triangulated category $\mathcal{T}$, we mean an object $G$ whose closure under shifts in both directions, extensions and passage to direct factors equals $\mathcal{T}$.

TheOREM 3.1 ([25, 7.6]). Let $\mathcal{T}$ be a (k-linear) algebraic triangulated category with split idempotents and a generator $G$. Then there is a structure of $A_{\infty}$-algebra on

$$
A=\bigoplus_{n \in \mathbf{Z}} \operatorname{Hom}_{\mathcal{T}}(G, G[n])
$$

such that $m_{1}=0, m_{2}$ is given by composition and that the functor

$$
\mathcal{T} \rightarrow \operatorname{Grmod}\left(A, m_{2}\right), U \mapsto \bigoplus_{n \in \mathbf{Z}} \operatorname{Hom}_{\mathcal{T}}(G, U[n])
$$

lifts to a triangle equivalence

$$
\mathcal{T} \rightarrow \operatorname{per}(A)
$$


Here Grmod denotes the category of graded right modules. For example, suppose that $\mathcal{T}$ is the bounded derived category of the category of coherent sheaves on projective $n$-space. Then, as Beilinson has shown [4 by 'resolving the diagonal', the object $G=\bigoplus_{i=0}^{n} O(-i)$ generates $\mathcal{T}$. Moreover, the algebra $A$ is concentrated in degree 0 and of finite dimension and finite global dimension. Then we obtain equivalences

$$
\mathrm{D}^{b}\left(\operatorname{coh} \mathbf{P}^{n}\right) \stackrel{\sim}{\rightarrow} \operatorname{per}(A) \leftarrow \mathrm{D}^{b}(\bmod A),
$$

where $\bmod A$ denotes the category of finite-dimensional $A$-modules.

Beautiful theorems on the existence of generators in triangulated categories of geometric origin are due to Bondal-Van den Bergh [5] and to Rouquier [35.

\section{The bar-cobar equivalence}

In this section, we present the interpretation of $A_{\infty}$-algebras as the fibrant objects in the model category of certain differential graded coalgebras following 25. For the reader's convenience we first recall the notion of model category.

4.1. Reminder on model categories. Model categories were invented by D. Quillen [33. A good modern introduction can be found in [7. A model category is a category $\mathcal{C}$ endowed with three classes of morphisms

- the class $\mathcal{W}$ whose elements are called weak equivalences and denoted by arrows with tilde $\stackrel{\sim}{\rightarrow}$,

- the class $\mathcal{F} i b$ whose elements are called fibrations and denoted by feathered arrows $\longrightarrow$,

- the class $\mathcal{C}$ of whose elements are called cofibrations and denoted by doubleheaded arrows $\longrightarrow$.

Each of these classes is supposed to be stable under compositions and to contain the identities. Moreover, the classes are supposed to satisfy the following axioms

MC1 The category $\mathcal{C}$ admits all finite limits and colimits (in particular, it admits an initial object $\emptyset$ and a terminal object $*$ ).

$\mathrm{MC} 2$ The class $\mathcal{W}$ is saturated, $i$. e. if two among $f, g$, and $f g$ belong to $\mathcal{W}$ so does the third.

MC3 The three classes $\mathcal{W}, \mathcal{F} i b$ and $\mathcal{C}$ of are stable under retracts in the category of morphisms of $\mathcal{C}$.

MC4 In each commutative square of solid arrows of the following two types
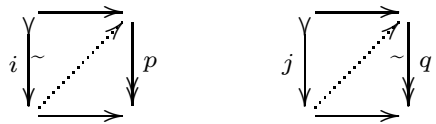

the dotted arrow exists so as to make the two triangles commutative.

MC5 Each morphism $f$ factors as $f=p i$, where $p$ is a fibration and $i$ a cofibration and a weak equivalence. It also factors as $f=q j$, where $q$ is a fibration and a weak equivalence and $j$ a cofibration.

Note that these axioms are selfdual. In the situation of $\mathrm{MC}$, one says that $p$ has the right lifting property with respect to $i$ and $i$ the left lifting propery with respect to $p$. It is not hard to see that if $\mathcal{C}$ is a model category, then the class $\mathcal{F} i b$ is determined by the class of trivial cofibrations $\mathcal{W} \cap \mathcal{C}$ of and the class $\mathcal{C} o f$ is determined by the class of trivial fibrations $\mathcal{W} \cap \mathcal{F} i b$. In fact, $\mathcal{F} i b$ consists of the morphisms with the right lifting property for all trivial cofibrations and $\mathcal{C}$ of of the morphisms with 
the left lifting property with respect to all trivial fibrations. A model category is left proper if any pushout of a weak equivalence along a cofibration is a weak equivalence.

As an example, consider the category $\mathcal{C}=\mathcal{C}^{+}(\operatorname{Mod} B)$ of left bounded complexes

$$
\ldots \rightarrow 0 \rightarrow \ldots \rightarrow X^{p} \rightarrow X^{p+1} \rightarrow \ldots
$$

of right modules over a ring $B$. We define $\mathcal{W}$ to be the class of quasi-isomorphisms, $\mathcal{C}$ of to be the class of morphisms $i: X \rightarrow Y$ such that each $i^{n}, n \in \mathbf{Z}$, is injective and $\mathcal{F} i b$ to be the class of morphisms $p: X \rightarrow Y$ such that each morphism $p^{n}$, $n \in \mathbf{Z}$, is surjective and has an injective kernel. It is not hard to show, $c f$. [14, that $\mathcal{C}$ is a model category. Note that in $\mathcal{C}$ the initial and the terminal object coincide with the complex of zero modules, that the morphism $0 \rightarrow X$ is always a cofibration and that the morphism $X \rightarrow 0$ is a fibration iff all components $X^{n}, n \in \mathbf{Z}$, are injective. Therefore, in this example, the first half of axiom MC5 corresponds to the existence of injective resolutions for complexes

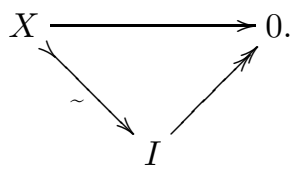

In an arbitrary model category $\mathcal{C}$, an object $X$ is fibrant if the morphism $X \rightarrow *$ is a fibration; an object $Y$ is cofibrant if the morphism $\emptyset \rightarrow Y$ is a cofibration.

Thus, in the above example, all complexes $X$ are cofibrant and a complex $Y$ is fibrant iff it has injective components.

For an arbitrary model category $\mathcal{C}$, the homotopy category $\mathrm{Ho}(\mathcal{C})$ is defined as the localization $\mathcal{C}\left[\mathcal{W}^{-1}\right]$ of the category $\mathcal{C}$ with respect to the class $\mathcal{W}$. Thus, it has the same objects as $\mathcal{C}$ and its morphisms are obtained from the morphisms of $\mathcal{C}$ by formally inverting all elements of $\mathcal{W}$. In the above example, the homotopy category equals the left bounded derived category $\mathrm{D}^{+}(\operatorname{Mod} B)$.

TheOREM 4.1. Let $\mathcal{C}$ be a model category and $\mathcal{C}_{c f}$ the full subcategory formed by the objects $X$ which are both fibrant and cofibrant. Then, for $X, Y \in \mathcal{C}_{c f}$, the canonical map

$$
\operatorname{Hom}_{\mathcal{C}}(X, Y) \rightarrow \operatorname{Hom}_{\mathrm{Ho}(\mathcal{C})}(X, Y)
$$

is surjective.

With the notations of the theorem, define two morphisms $f, g$ from $X$ to $Y$ to be homotopic if their images in the homotopy category coincide. Is is then clear that the canonical functor

$$
\mathcal{C}_{c f} / \text { homotopy } \rightarrow \mathrm{Ho}(\mathcal{C})
$$

is an equivalence.

In our example, a complex $X$ belongs to $\mathcal{C}_{c f}$ iff it has injective components. Two morphisms $f, g$ between such complexes are then homotopic in the sense of the model category iff they are homotopic in the classical sense, $i$. $e$. there is a $B$-linear morphism $r: X \rightarrow Y$ homogeneous of degree -1 such that

$$
f-g=d \circ r+r \circ d .
$$

Finally, the above equivalence specializes to the classical equivalence

$$
\mathcal{C}^{+}(\operatorname{Inj} B) / \text { homotopy } \stackrel{\sim}{\rightarrow} \mathrm{D}^{+}(\operatorname{Mod} B),
$$


where $\operatorname{lnj} B$ denotes the full subcategory of injective $B$-modules.

In a model category $\mathcal{C}$, an object $I$ is minimal if it is fibrant and cofibrant and for all endomorphisms $f$ of $I$ in $\mathcal{C}$, we have

$f$ is invertible in $\mathcal{C} \Leftrightarrow f$ becomes invertible in $\mathrm{Ho}(\mathcal{C})$.

A minimal model for $X \in \mathcal{C}$ is an isomorphism

$$
X \stackrel{\sim}{\rightarrow} I
$$

of $\mathrm{Ho}(\mathcal{C})$ such that $I$ is minimal.

In our example, assume that $X=M$ is a $B$-module, $i$. e. a complex concentrated in degree 0. Suppose that we have an injective resolution

$$
0 \rightarrow M \rightarrow I^{0} \rightarrow I^{1} \rightarrow \ldots
$$

Such a resolution gives rise to an isomorphism $M \stackrel{\sim}{\rightarrow} I$ in the derived category, i. $e$. $\mathrm{Ho}(\mathcal{C})$. This is a minimal model for $M$ iff the above resolution is a minimal injective resolution.

4.2. Algebras. Let $k$ be a field. Let Alg be the category of augmented $d g$ algebras, $i$. e. unital associative dg algebras $A$ endowed with a morphism

$$
\varepsilon: A \rightarrow k
$$

of unital dg algebras. In Alg, define $\mathcal{W}$ to be the class of quasi-isomorphisms and $\mathcal{F} i b$ to be the class of surjective morphisms. Let $\mathcal{C}$ of be the class of morphisms $i$ such that in each commutative square of solid arrows in Alg

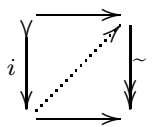

there is a dotted arrow which makes the two triangles commutative. Note that the algebra $k$ is both the initial and the terminal object of Alg.

THEOREM 4.2 (Hinich [13). The category Alg endowed with the above classes $\mathcal{W}, \mathcal{F} i b$ and $\mathcal{C}$ of is a model category. All its objects are fibrant.

Below, we will give a description of the cofibrant objects of Alg. We will see that if an object of Alg is cofibrant, then its underlying graded coalgebra is free. However, one can show that this condition is not sufficient. One can also show that not all objects $A \in$ Alg admit minimal models.

4.3. Coalgebras and the bar/cobar constructions. Let $\mathrm{Cog}^{\prime}$ be the category of augmented dg coalgebras. Thus, an object of $\operatorname{Cog}^{\prime}$ is a complex $C$ endowed with a coassociative comultiplication

$$
\Delta: C \rightarrow C \otimes C
$$

for which $d$ is a coderivation, $i$. $e$.

$$
\Delta \circ d=(d \otimes \mathbf{1}+\mathbf{1} \otimes d) \circ \Delta,
$$

such that $\Delta$ admits a counit $\eta: C \rightarrow k$ and a (co-)augmentation $\varepsilon: k \rightarrow C, i$. e. a counital coalgebra morphism such that $\eta \varepsilon=\mathbf{1}_{k}$. For example, if $V$ is a complex, then we let $T^{c}(V)$ be the tensor coalgebra on $V$ : Its underlying complex is

$$
\bigoplus_{n \geq 0} V^{\otimes n}
$$


and its comultiplication 'separates tensors':

$$
\Delta\left(v_{1}, \ldots, v_{n}\right)=\sum_{i=0}^{n}\left(v_{1}, \ldots, v_{i}\right) \otimes\left(v_{i+1}, \ldots, v_{n}\right),
$$

where $\left(v_{1}, \ldots, v_{n}\right)$ stands for $v_{1} \otimes \ldots \otimes v_{n}$ and the empty parentheses () are to be interpreted as 1 . Then $T^{c}(V)$ is endowed with a canonical projection

$$
T^{c}(V) \rightarrow V
$$

dual to the inclusion $V \rightarrow T(V)$ of $V$ into the tensor algebra, but in the category $\mathrm{Cog}^{\prime}$, the coalgebra $T^{c}(V)$ does not have the universal property dual to that of the tensor algebra: it is not cofree on $V$. To resolve this technical problem is one of the motivations for replacing $\operatorname{Cog}^{\prime}$ by a smaller category, namely that of cocomplete augmented dg coalgebras. A coalgebra $C \in \mathrm{Cog}^{\prime}$ is cocomplete if it is the union of the kernels of the maps

$$
C \rightarrow C^{\otimes n} \rightarrow(C / k)^{\otimes n}, n \geq 2,
$$

which are the compositions of the canonical projection with the iterated comultiplications. Define Cog to be the full subcategory of $\mathrm{Cog}^{\prime}$ whose objects are the cocomplete coalgebras $C \in \mathrm{Cog}^{\prime}$. Then it is not hard to show that $T^{c}(V)$ is cocomplete and that it is cofree on $V$ in the category $\operatorname{Cog}$.

For a $\operatorname{dg}$ coalgebra $C$ and a $\operatorname{dg}$ algebra $A$, consider the complex

$$
\operatorname{Hom}_{k}{ }_{k}(C, A)
$$

whose $n$th component is the space of homogeneous $k$-linear maps $f: C \rightarrow A$ of degree $n$ and whose differential maps $f$ to

$$
d \circ f-(-1)^{n} f \circ d .
$$

This complex becomes a dg algebra for the convolution defined by

$$
f * g=\mu \circ(f \otimes g) \circ \Delta .
$$

Define a homogeneous $k$-linear map $\tau: C \rightarrow A$ to be a twisting cochain if it is homogeneous of degree 1 and satisfies

$$
d(\tau)+\tau * \tau=0 \text { and } \varepsilon \circ \tau \circ \varepsilon=0 .
$$

Let $\operatorname{Tw}(C, A)$ be the set of twisting cochains. Then it is not hard to show that for a given $A \in \mathrm{Alg}$, the functor

$$
\operatorname{Cog} \rightarrow \text { Sets }, C \mapsto \operatorname{Tw}(C, A)
$$

is representable. The bar construction $B A$ is by definition a representative. It is endowed with the universal twisting cochain $\tau_{0}$ which corresponds to the identity of $B A$ under the bijection

$$
\operatorname{Tw}(B A, A)=\operatorname{Hom}_{\operatorname{cog}}(B A, B A) .
$$

One checks that we have $B A=T^{c}(S A)$ endowed with a differential which takes into account the differential of $A$ and its multiplication. The universal twisting cochain is the canonical projection $B A \rightarrow A$.

Dually, one shows that for a coalgebra $C$, the functor

$$
\text { Alg } \rightarrow \text { Sets }, A \mapsto \operatorname{Tw}(C, A)
$$

is corepresentable. The cobar construction $\Omega(C)$ is a corepresentative. One has $\Omega C=T\left(S^{-1} C\right)$ and the differential of $\Omega C$ takes into account the differential of $C$ 
and the comultiplication. The universal twisting cochain is the canonical inclusion $C \rightarrow \Omega C$.

It is clear from these constructions that for $A \in \mathrm{Alg}$ and $C \in \mathrm{Cog}$, we have

$$
\operatorname{Hom}_{\mathrm{Alg}}(\Omega(C), A)=\operatorname{Tw}(C, A)=\operatorname{Hom}_{\mathrm{Cog}}(C, B(A))
$$

so that $\Omega$ is left adjoint to $B$. If $A$ is an ordinary augmented algebra (concentrated in degree 0 ) and $C$ an ordinary augmented coalgebra, one checks that

$$
H_{*}(B A)=\operatorname{Tor}_{*}^{A}(k, k) \text { and } H^{*}(\Omega C)=\operatorname{Cotor}_{C}^{*}(k, k) .
$$

In Cog, we define $\mathcal{W}$ to be the class of morphisms $f: C \rightarrow D$ such that $\Omega(f)$ is a quasi-isomorphism; we define $\mathcal{C}$ of to be the class of injective morphisms and $\mathcal{F} i b$ to be the class of morphisms $p$ such that in each commutative square of solid arrows in $\mathrm{Cog}$

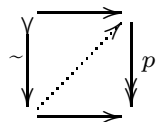

there is a dotted arrow which makes the two triangles commutative.

TheOREM 4.3 (Lefèvre [25, 1.3]). a) The category $\operatorname{Cog}$ endowed with the above classes $\mathcal{W}, \mathcal{C}$ of and $\mathcal{F} i b$ is a model category. The functors $B$ and $\Omega$ induce quasi-inverse equivalences

$$
\mathrm{Ho}(\mathrm{Alg}) \stackrel{\sim}{\rightarrow} \mathrm{Ho}(\mathrm{Cog}) .
$$

Moreover, $B$ preserves fibrations and trivial fibrations and $\Omega$ preserves cofibrations and trivial cofibrations.

b) All objects of Alg are fibrant. An object of Alg is cofibrant iff it is a retract of $\Omega C$ for some $C$ in $\operatorname{Cog}$. All objects of $\operatorname{Cog}$ are cofibrant. An object is fibrant iff its underlying graded coalgebra is a tensor coalgebra.

c) Two morphisms $f, g: A \rightarrow A^{\prime}$ between fibrant-cofibrant objects of Alg are homotopic iff there is a $k$-linear map $h: A \rightarrow A^{\prime}$ homogeneous of degree -1 such that

$\varepsilon \circ h=0$ and $h \circ m_{A}=m_{A^{\prime}} \circ(f \otimes h+h \otimes g)$ and $f-g=d \circ h+h \circ d$.

The dual statement holds for morphisms between fibrant-cofibrant objects of Cog.

Notice that Alg and Cog behave similarly in many respects but that the descriptions of their fibrant-cofibrant objects are quite different. Below, in section 4.5 we give an example of a non cofibrant object of Alg whose underlying graded algebra is free. Hinich [13 describes the cofibrations of Alg as 'transfinite cell attachments'.

4.4. The link with $A_{\infty}$-algebras and minimal models. An augmented $A_{\infty}$-algebra is a strictly unital $A_{\infty}$-algebra endowed with a strict unit-preserving morphism $\varepsilon: A \rightarrow k$. Such an algebra decomposes as $A=k \oplus \bar{A}$ and the functor $A \mapsto \bar{A}$ yields an equivalence between the category of augmented $A_{\infty}$-algebras and the category of non unital $A_{\infty}$-algebras.

For a coalgebra $C \in \operatorname{Cog}$ and an augmented $A_{\infty}$-algebra $A$, the complex $\operatorname{Hom}^{\bullet}{ }_{k}(C, A)$ becomes an augmented $A_{\infty}$-algebra for the convolution operations

$$
b_{n}\left(f_{1}, \ldots, f_{n}\right)=b_{n}^{A} \circ\left(f_{1} \otimes \cdots \otimes f_{n}\right) \circ \Delta^{(n)},
$$


where $\Delta^{(n)}$ is the iterate of $\Delta$ taking values in $C^{\otimes n}$. Define $\operatorname{Tw}_{\infty}(C, A)$ to be the set of solutions $\tau$ of the equation

$$
\sum_{n \geq 1} b_{n}(\tau, \ldots, \tau)=0
$$

Note that the sum yields a well-defined map $C \rightarrow A$ thanks to the fact that $C$ is cocomplete. Then the functor

$$
\operatorname{Cog} \rightarrow \text { Sets }, C \rightarrow \mathrm{Tw}_{\infty}(C, A)
$$

is representable and we denote by $B_{\infty} A$ a representative. It is not hard to check that $B_{\infty} A$ is $T^{c}(S A)$ endowed with the unique coderivation whose composition with the projection $B A \rightarrow S A$ has the components

$$
b_{n}:(S A)^{\otimes n} \rightarrow S A, n \geq 1 .
$$

In fact, if $V$ is a graded vector space, then there is a bijection between $A_{\infty}$-structures on $V$ and coalgebra differentials on $T S V$. Moreover, if $A$ and $A^{\prime}$ are $A_{\infty}$-algebras, then the $A_{\infty}$-morphisms $f: A \rightarrow A^{\prime}$ are in canonical bijection with the morphisms $B_{\infty} A \rightarrow B_{\infty} A^{\prime}$ of augmented dg coalgebras.

Theorem $4.4([\mathbf{2 5}, 1.3])$ a) An object of Cog is fibrant-cofibrant iff it is isomorphic to $B_{\infty} A$ for some $A_{\infty}$-algebra $A$.

b) Each object $C$ of $\operatorname{Cog}$ admits a minimal model and, if $A$ is an $A_{\infty}$-algebra and $A_{\text {min }}$ its minimal model, then $B_{\infty}\left(A_{\text {min }}\right)$ is the minimal model of $B_{\infty} A$.

It is not hard to see that an $A_{\infty}$-quasi-isomorphism $f: A \rightarrow A^{\prime}$ between $A_{\infty^{-}}$ algebras yields a weak equivalence $B_{\infty} A \rightarrow B_{\infty} A^{\prime}$. Since both of these coalgebras are fibrant-cofibrant, such a weak equivalence has an inverse up to homotopy. This yields that $f$ has an inverse up to $A_{\infty}$-homotopy. The theorem also yields a good interpretation of the notion of minimality for $A_{\infty}$-algebras. The following square summarizes the situation. We denote the category of augmented $A_{\infty}$-algebras by $\mathrm{Alg}_{\infty}$.

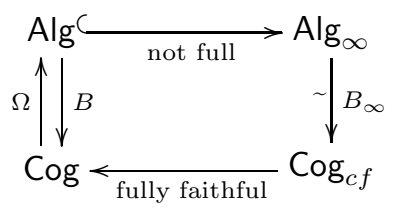

If we pass to the homotopy categories (where homotopy means localization for Alg and Cog and passage to quotient categories for $\mathrm{Alg}_{\infty}$ and $\operatorname{Cog}_{c f}$ ), then all the arrows of the diagram become equivalences. Thus we obtain four descriptions of the localization of the category of dg algebras with respect to all quasi-isomorphisms. In particular, $A_{\infty}$-algebras do not yield 'new homotopy types' of algebras but a new description of the existing types.

4.5. A non cofibrant dg algebra. Here is an example of a dg algebra $A \in$ Alg which is free as a graded algebra but not cofibrant: Take $A=T V$, where $V=k$ is concentrated in degree 1 . Endow $A$ with the unique differential whose restriction to $V \subset T V$ is

$$
V=k \stackrel{\sim}{\rightarrow} k \otimes k=V^{\otimes 2} \subset T V .
$$

Then clearly $A$ is quasi-isomorphic to its subalgebra $k$, which is fibrant-cofibrant. If $A$ was fibrant-cofibrant as well, then the inclusion $k \rightarrow T V$ should admit a left 
inverse up to homotopy in the sense of c) in theorem 4.3 But there are no nonzero maps $h: A \rightarrow k$ of degree -1 such that $\varepsilon \circ h=0$. Therefore, $A$ cannot be fibrant-cofibrant. Note that $A$ is the cobar construction on a non cocomplete $\mathrm{dg}$ coalgebra.

4.6. Modules and comodules. Let $A$ be an augmented dg algebra and $C$ a cocomplete augmented dg coalgebra. Let $\tau: C \rightarrow A$ be a twisting cochain. For a dg right $A$-module $L$, we endow the $C$-comodule $L \otimes C$ with the differential defined by

$$
d=d_{L} \otimes \mathbf{1}_{C}+\mathbf{1}_{L} \otimes d_{C}+\left(\mu \otimes \mathbf{1}_{C}\right)\left(\mathbf{1}_{L} \otimes \tau \otimes \mathbf{1}_{C}\right)\left(\mathbf{1}_{L} \otimes \Delta\right),
$$

where $\Delta: C \rightarrow C \otimes C$ is the comultiplication of $C$ and $\mu: L \otimes A \rightarrow L$ the multiplication of $L$. We write $L \otimes_{\tau} C$ for the resulting $\operatorname{dg} C$-comodule. Similarly, if $M$ is a $\operatorname{dg}$ right $C$-comodule, we define a differential on $M \otimes A$ by

$$
d=d_{M} \otimes \mathbf{1}_{A}+\mathbf{1}_{M} \otimes d_{A}+\left(\mathbf{1}_{M} \otimes \mu_{A}\right)\left(\mathbf{1}_{M} \otimes \tau \otimes \mathbf{1}_{A}\right)\left(\delta_{M} \otimes \mathbf{1}_{A}\right) .
$$

We denote the resulting dg $A$-module by $M \otimes_{\tau} A$.

Let us denote by Mod $A$ the category of right $\operatorname{dg} A$-modules and by $\operatorname{Comc} C$ the category of $\operatorname{dg} C$-comodules $M$ which are cocomplete, i. e. $M$ is the union of the kernels of the maps induced by iterated comultiplications

$$
M \rightarrow M \otimes \bar{C}^{\otimes n}, n \geq 2 .
$$

Then the pair

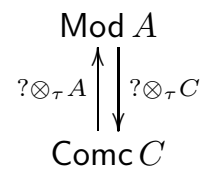

is a pair of adjoint functors ( $c f$. Lemme 2.2.1.2 of [25). From now on, we suppose that $\tau$ is acyclic, i. e. that the following equivalent (cf. Proposition 2.2.4.1 of [25]) conditions hold

(i) The adjunction morphism $M \otimes_{\tau} C \otimes_{\tau} A \rightarrow M$ is a quasi-isomorphism for each dg $A$-module $M$.

(ii) The adjunction morphism $A \otimes_{\tau} C \otimes_{\tau} A \rightarrow A$ is a quasi-isomorphism.

(iii) The morphism $B C \rightarrow A$ induced by $\tau$ is a quasi-isomorphism.

(iv) The morphism $C \rightarrow \Omega A$ induced by $\tau$ is a weak equivalence.

If follows from 13 that the category Mod $A$ admits a unique structure of model category whose weak equivalences are the quasi-isomorphisms and whose fibrations are the surjective morphisms. We write $\mathrm{D}(A)$ for the localization of $\operatorname{Mod} A$ with respect to the class of quasi-isomorphisms.

Theorem 4.5 (Théorème 2.2.2.2 of $[\mathbf{2 5}$ ). $\quad$ a) The category Comc $C$ admits a unique structure of model category whose weak equivalences are the morphisms $f$ such that $f \otimes_{\tau} A$ is a quasi-isomorphism and whose cofibrations are the injetive morphisms. We write $\mathrm{D}(C)$ for the localization of Comc $C$ with respect to the class of weak equivalences.

b) The functors? $\otimes_{\tau} C$ and $? \otimes_{\tau} A$ induce quasi-inverse equivalences

$$
\mathrm{D}(A) \stackrel{\sim}{\rightarrow} \mathrm{D}(C) .
$$


4.7. Example: Koszul algebras. Let $V$ be a vector space and $R \subset V \otimes V$ a subspace such that the algebra $A=T V /(R)$ is Koszul [2], $i$. e. the $A$-module $k$ admits a minimal projective resolution $P \rightarrow k$ in the category of graded $A$-modules such that $P_{i}$ is generated in degree $i$ for all $i \geq 0$. Let $C$ be the subspace of the tensor coalgebra $T^{c}(V)$ whose $n$th component is $k$ for $n=0, V$ for $n=1$ and

$$
\bigcap_{p+2+q=n} V^{\otimes p} \otimes R \otimes V^{\otimes q}
$$

for $n \geq 2$. Then clearly $C$ is a subcoalgebra. We endow $C$ with the grading such that $V$ is in degree -1 and with the vanishing differential. We consider $A$ as a $\operatorname{dg}$ algebra concentrated in degree 0 . We let $\tau: A \rightarrow C$ be given by the composition

$$
C \rightarrow V \rightarrow A
$$

of the inclusion with the projection. Clearly, $\tau$ is a twisting cochain. Then the complex

$$
A \otimes_{\tau} C \otimes_{\tau} A
$$

is the Koszul resolution of the bimodule $A$. Thus, the twisting cochain $\tau$ is acyclic and we obtain an equivalence of categories

$$
? \otimes_{\tau} C: \mathrm{D}(A) \rightarrow \mathrm{D}(C)
$$

which takes $A$ to $k$ (weakly equivalent to $A \otimes_{\tau} C$ ) and $k$ to $C$. An analogous equivalence can be constructed between suitable (Adams-) graded versions of the categories $\mathrm{D}(A)$ and $\mathrm{D}(C)$. We refer to $[\underline{8}$ for related developments.

4.8. Link with $A_{\infty}$-modules. Let $A$ be an augmented $A_{\infty}$-algebra. Let $C=B_{\infty} A$. The adjunction morphism

$$
B_{\infty} A=C \rightarrow B \Omega C=B_{\infty}(\Omega C)
$$

corresponds to a canonical $A_{\infty}$-morphism $A \rightarrow \Omega B_{\infty} A$, which is a quasi-isomorphism and which is universal among the $A_{\infty}$-morphisms from $A$ to a dg algebra (Lemme 2.3.4.3 of [25]). Let us put $U(A)=\Omega B_{\infty} A$. Thus we have a canonical acyclic twisting cochain $\tau: B_{\infty}(A) \rightarrow U(A)$. By theorem 4.5 we have an equivalence

$$
\mathrm{D}(U(A)) \stackrel{\sim}{\rightarrow} \mathrm{D}\left(B_{\infty}(A)\right) .
$$

Both of these categories can be regarded as the (unbounded) derived category of the $A_{\infty}$-algebra $A$. The link with $A_{\infty}$-modules is the following: Let $\operatorname{Mod}_{\infty} A$ be the category of $A_{\infty}$-modules over $A$ which are strictly unital (i. e. $b_{n}^{M}, n \geq 2$, vanishes as soon as one of the arguments is 1 ) and whose morphisms are strictly unital (i.e. $f_{n}, n \geq 2$, vanishes as soon as one of the arguments is 1 ). This category is isomorphic to the category of all $A_{\infty}$-modules with all $A_{\infty}$-morphisms over the reduction $\bar{A}$. By restriction along the $A_{\infty}$-morphism $A \rightarrow U(A)$, each dg module over $U(A)$ yields an $A_{\infty}$-module in $\operatorname{Mod}_{\infty} A$. However, the resulting functor

$$
\operatorname{Mod} U(A) \rightarrow \operatorname{Mod}_{\infty} A
$$

is not full (its image only contains strict morphisms).

Let $M$ be in $\operatorname{Mod}_{\infty} A$. The datum of its strictly unital $A_{\infty}$-module structure over $A$ is equivalent to the datum of an arbitrary $A_{\infty}$-module structure over $\bar{A}$. This in turn is equivalent to giving a comodule differential on the induced comodule 
$M \otimes B_{\infty} A$. We write $B_{\infty} M$ for the induced comodule endowed with the differential corresponding to a given $A_{\infty}$-module structure on $M$. Thus we obtain a functor

$$
\operatorname{Mod}_{\infty} A \rightarrow \operatorname{Comc} B_{\infty}(A), M \rightarrow B_{\infty} M .
$$

It is not hard to see that this functor is in fact fully faithful. We define two morphisms of $\operatorname{Mod}_{\infty} A$ to be homotopic if they become homotopic in Comc $B_{\infty}(A)$.

Proposition 4.6. $\quad$ a) The functor $M \mapsto B_{\infty} M$ induces an equivalence onto the full subcategory (Comc $\left.B_{\infty} A\right)_{c f}$ of fibrant-cofibrant objects of Comc $B_{\infty}(A)$.

b) The functor $M \mapsto B_{\infty} M$ induces an equivalence

$$
\left(\operatorname{Mod}_{\infty} A\right) / \text { homotopy } \stackrel{\sim}{\rightarrow} \mathrm{D}(C) .
$$

Proof. a) In Proposition 2.4.1.3 of [25], it is shown that $B_{\infty} M$ is fibrant (and cofibrant) in Comc $B_{\infty} A$ and that each fibrant object is a direct factor of an object $M \otimes_{\tau} B_{\infty} A$ for some $U(A)$-module $M$. It remains to be shown that such a direct factor is of the form $B_{\infty} M^{\prime}$ for some $M^{\prime} \in \operatorname{Mod}_{\infty} A$. Put $C=B_{\infty} A$. We will use that a morphism $g: V \otimes C \rightarrow W \otimes C$ of induced $C$-comodules is invertible iff it induces an invertible morphism in the spaces of primitive elements

$$
V=(V \otimes C)_{[1]}=\operatorname{ker}\left(V \otimes C \stackrel{\mathbf{1}_{V} \otimes \Delta-\mathbf{1}_{V} \otimes \mathbf{1}_{V} \otimes \eta}{\longrightarrow} V \otimes C \otimes C\right) .
$$

If $f$ is an idempotent endomorphism of $M \otimes_{\tau} C$, it induces an idempotent endomorphism of graded vector spaces in $M=\left(M \otimes_{\tau} C\right)_{[1]}$. Let $M=M^{\prime} \oplus M^{\prime \prime}$ be the corresponding decomposition in the category of graded vector spaces. Then we have $M \otimes C=\left(M^{\prime} \otimes C\right) \oplus\left(M^{\prime \prime} \otimes C\right)$ as $C$-comodules and in the corresponding decomposition

$$
f=\left[\begin{array}{ll}
f_{11} & f_{12} \\
f_{21} & f_{22}
\end{array}\right]
$$

the diagonal entries are invertible. It follows that the image $I$ of $f$ is isomorphic to $M^{\prime} \otimes C$ as a $C$-comodule. The differential of $M \otimes_{\tau} C$ yields a comodule differential on $I \stackrel{\sim}{\rightarrow} M^{\prime} \otimes C$ and thus an $A_{\infty}$-module structure on $M^{\prime}$. We have $I \stackrel{\sim}{\rightarrow} B_{\infty} M^{\prime}$. Point b) is proved in Lemme 2.4.2.3 of [25].

To sum up, we have the following diagram

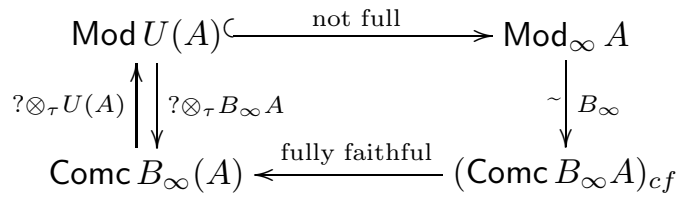

All functors induce equivalences in the localizations (for the two categories on the left) respectively the quotients by the homotopy relations (for the two categories on the right).

4.9. The derived category of a non augmented $A_{\infty}$-algebra. Let $A$ be a (non augmented) $A_{\infty}$-algebra. Let $A^{+}=A \oplus k$ be the augmented $A_{\infty}$-algebra obtained by adjoining $k$. The augmentation $A^{+} \rightarrow k$ yields a functor

$$
\operatorname{Mod}_{\infty} A^{+} \rightarrow \operatorname{Mod}_{\infty} k
$$


which passes to the derived categories, $c f$. section 4.1.1 of [25]. By definition, the (unbounded) derived category $\mathrm{D}_{\infty}(A)$ is the kernel of the functor

$$
\mathrm{D}_{\infty}\left(A^{+}\right) \rightarrow \mathrm{D}_{\infty}(k) .
$$

One can show that a strictly unital $A_{\infty}$-module $M$ over $A^{+}$is in the kernel iff $B_{\infty} M$ is acyclic (Remarque 4.1.3.5 of [25]).

Now suppose that $A$ is homologically unital, i. e. $H^{*}(A)$ endowed with the multiplication induced by $m_{2}$ is unital. Then $M$ belongs to the kernel iff $M$ is homologically unital, i. e. $H^{*}(M)$ is a unital $H^{*}(A)$-module, cf. Lemme 4.1.3.7 of 25. Moreover, in this case $\mathrm{D}_{\infty}(A)$ is a compactly generated triangulated category and has the free $A$-module of rank one as a compact generator. Indeed, $A^{+}$is quasi-isomorphic to $U\left(A^{+}\right)$and one checks that if $B=k \oplus \bar{B}$ is an augmented dg algebra with homologically unital augmentation ideal $\bar{B}$, then $\bar{B}$ is a compact generator for the kernel of $\mathrm{D}(B) \rightarrow \mathrm{D}(k)$.

\section{A conceptual construction of $A_{\infty}$-functor categories}

The notion of $A_{\infty}$-algebra naturally generalizes to that of $A_{\infty}$-category. It has been known for some time, $c f$. $\mathbf{2 2}$, that for two $A_{\infty}$-categories $\mathcal{A}, \mathcal{B}$, there is a natural $A_{\infty}$-category $\operatorname{Fun}_{\infty}(\mathcal{A}, \mathcal{B})$ and for three $A_{\infty}$-categories, a natural ' $A_{\infty}$ bifunctor'

$$
\operatorname{Fun}_{\infty}(\mathcal{B}, \mathcal{C}) \times \operatorname{Fun}_{\infty}(\mathcal{A}, \mathcal{B}) \rightarrow \operatorname{Fun}_{\infty}(\mathcal{A}, \mathcal{C})
$$

One can use this composition bifunctor to give a natural construction for the bialgebra structure of [1] 11] on the bar construction $B C$ of the Hochschild complex $C=C(A, A)$ of an algebra $A, c f$. section 5.7

The $A_{\infty}$-category $\operatorname{Fun}_{\infty}(\mathcal{A}, \mathcal{B})$ was constructed in [25] using twists of $A_{\infty}$ structures. Here, we further develop an idea of V. Lyubashenko's $[\mathbf{2 7}$ and interpret $\operatorname{Fun}_{\infty}(\mathcal{A}, \mathcal{B})$ as an internal Hom-object in a tensor category, namely the category of cocomplete augmented dg cocategories. The main difference with V. Lyubashenko's approach is that he only considers the tensor subcategory generated by all cofree cocategories.

5.1. Augmented $k$-quivers. Let $k$ be a commutative ring. A $k$-quiver $V$ consists of a set of objects obj $(V)$ and of $k$-modules $V(x, y)$, for all objects $x, y$ of $V$. A morphism of $k$-quivers $F: V \rightarrow W$ consists of a map $F: \operatorname{obj}(V) \rightarrow \operatorname{obj}(W)$ and of maps

$$
F_{x, y}: V(x, y) \rightarrow W(F x, F y),
$$

for all objects $x, y$ of $V$. For each set $S$, we have the discrete $k$-quiver $k S$ with $\operatorname{obj}(k S)=S,(k S)(x, x)=k \mathbf{1}_{x}$ and $(k S)(x, y)=0$ for all objects $x \neq y$ of $k S$. An augmented $k$-quiver is a $k$-quiver $V$ endowed with morphisms

$$
k \operatorname{obj}(V) \stackrel{\eta}{\rightarrow} V \stackrel{\varepsilon}{\rightarrow} k \operatorname{obj}(V)
$$

whose composition is the identity of $k \operatorname{obj}(V)$. A morphism of augmented $k$-quivers is a morphism of the underlying quivers compatible with the morphisms $\eta$ and $\varepsilon$. The tensor product of two augmented $k$-quivers $V$ and $W$ is the naturally augmented $k$-quiver $V \otimes W$ whose set of objects is $\operatorname{obj}(V) \times \operatorname{obj}(W)$ and such that

$$
(V \otimes W)\left(\left(x, x^{\prime}\right),\left(y, y^{\prime}\right)\right)=V(x, y) \otimes W\left(x^{\prime}, y^{\prime}\right) .
$$

The unit augmented $k$-quiver $E$ has one object $*$ and $E(*, *)=k$. It is endowed with the identity morphisms $\eta$ and $\varepsilon$. Clearly, we have natural isomorphisms $E \otimes V \stackrel{\sim}{\rightarrow}$ 
$V \leftarrow V \otimes E$ for each augmented $k$-quiver $V$. For two augmented $k$-quivers $V$, $W$, we define $\underline{\operatorname{Hom}}_{q}(V, W)$ to be the $k$-quiver whose objects are the morphisms of augmented $k$-quivers $f: V \rightarrow W$ and such that, for $f \neq g$, we have

$$
\underline{\operatorname{Hom}}_{q}(V, W)(f, g)=\prod_{x, y \in \operatorname{obj}(V)} \operatorname{Hom}_{k}(V(x, y), W(f x, g y)),
$$

and for $f=g$, the $k$-module $\underline{\operatorname{Hom}}_{q}(V, W)(f, f)$ is formed by the families $\left(\varphi_{x, y}\right)$ of

$$
\prod_{x, y \in \operatorname{obj}(V)} \operatorname{Hom}_{k}(V(x, y), W(f x, f y))
$$

such that, for all $x, y$ in obj $(V)$, we have

$$
\varepsilon_{f(x), g(y)} \circ \varphi_{x, y}=\varepsilon_{x, y}
$$

and the scalar

$$
\varepsilon_{f x, f x} \circ \varphi_{x, x} \circ \eta_{x, x}
$$

is independent of $x \in \operatorname{obj}(V)$. We endow $\underline{\operatorname{Hom}}_{q}(V, W)$ with the morphism $\varepsilon$ which takes a family $\left(\varphi_{x, y}\right)$ to this scalar and with the morphism $\eta$, which takes $\mathbf{1}_{f}$ to the family

$$
\varphi_{x, y}: v \mapsto f(v) .
$$

Clearly we obtain an augmented $k$-quiver $\underline{\operatorname{Hom}}_{q}(V, W)$. For example, we have $\underline{\operatorname{Hom}}_{q}(E, W) \stackrel{\sim}{\rightarrow} W$.

A graded $k$-quiver (resp. a differential graded $k$-quiver) is a $k$-quiver $V$ such that $V(x, y)$ is a $\mathbf{Z}$-graded $k$-module (resp. a cochain complex of $k$-modules) for all objects $x, y$ of of $V$. One defines the tensor product and the functor $\underline{H o m}_{q}$ for graded and differential graded augmented $k$-quivers analogously.

LEMMA 5.1. The category of augmented $k$-quivers is monoidal with unit object $E$ and $\underline{\mathrm{Hom}}_{q}$ is an internal Hom-functor, i.e. we have a functorial bijection

$$
\operatorname{Hom}(U \otimes V, W) \stackrel{\sim}{\rightarrow} \operatorname{Hom}\left(U, \underline{\operatorname{Hom}}_{q}(V, W)\right)
$$

for all augmented $k$-quivers $U, V, W$. The same assertions hold for the categories of graded and that of differential graded augmented $k$-quivers.

For an augmented $k$-quiver $V$, we denote by $\bar{V}$ the reduction of $V$, i.e. the $k$-quiver with the same objects, and with

$$
\bar{V}(x, y)= \begin{cases}V(x, y) & \text { if } x \neq y \\ V(x, x) / \operatorname{im} \eta_{x, x} & \text { if } x=y .\end{cases}
$$

The reduction functor is an equivalence from the category of augmented $k$-quivers to that of $k$-quivers but this equivalence is not monoidal for the naive tensor product of $k$-quivers.

The augmented $k$-quivers with one object form a full monoidal subcategory of the category of augmented $k$-quivers but this subcategory does not admit an internal Hom-functor. 
5.2. Augmented cocomplete cocategories. A cocategory is a $k$-quiver $C$ endowed with $k$-linear maps

$$
\Delta: C(x, y) \rightarrow \bigoplus_{z \in \mathrm{obj}(C)} C(z, y) \otimes C(x, z)
$$

for all objects $x, y$ of $C$ such that the natural coassociativity condition holds. It is cocomplete if each $f \in C(x, y)$ lies in the kernel of a sufficiently high iterate of $\Delta$. It is counital if it is endowed with a morphism of $k$-quivers $\eta: C \rightarrow k$ obj $C$ such that the two counit equations hold. An augmented cocategory is a counital cocategory $C$ endowed with a morphism of counital cocategories $\varepsilon: k \operatorname{obj}(C) \rightarrow C$ such that the composition of $\eta$ with $\varepsilon$ is the identity of $k$ obj $(C)$. Then it is in particular an augmented $k$-quiver and the reduced quiver $\bar{C}$ becomes a cocategory. The augmented cocategory $C$ is cocomplete if its reduction $\bar{C}$ is cocomplete. These definitions admit obvious graded and differential graded variants.

Clearly the unit object $E$ of the category of augmented $k$-quivers admits a unique structure of (cocomplete) augmented cocategory. The tensor product $C_{1} \otimes$ $C_{2}$ of the $k$-quivers underlying two cocomplete augmented cocategories admits a natural structure of cocomplete augmented cocategory. For this tensor product, the category of cocomplete augmented cocategories becomes a monoidal category with unit object $E$.

Let $V$ be an augmented $k$-quiver. The tensor cocategory $T V$ has the same objects as $V$; and for any two objects $x, y$ of $T V$, the $k$-module $(T V)(x, y)$ is the direct sum of $V(x, y)$ with the tensor products

$$
\bar{V}\left(z_{n}, y\right) \otimes \bar{V}\left(z_{n-1}, z_{n}\right) \otimes \cdots \otimes \bar{V}\left(x, z_{1}\right)
$$

where $n \geq 1$ and $\left(z_{1}, \ldots, z_{n}\right)$ runs through all sequences of objects of $V$. For an element $\left(f_{n}, \ldots, f_{1}\right), n \geq 1$, of $\overline{T V}(x, y)$, one puts

$$
\begin{aligned}
\Delta\left(f_{n}, \ldots, f_{1}\right)= & \eta_{y, y} \otimes\left(f_{n}, \ldots, f_{1}\right)+\sum_{i=1}^{n}\left(f_{n}, \ldots, f_{i+1}\right) \otimes\left(f_{i}, \ldots, f_{1}\right) \\
& +\left(f_{n}, \ldots, f_{1}\right) \otimes \eta_{x, x} .
\end{aligned}
$$

The counit (resp. the coaugmentation) of $T V$ are obtained by composing the corresponding morphisms $V \rightarrow k$ obj $(V)$ (resp. $k$ obj $(V) \rightarrow V$ ) with the projection $p r_{1}: T V \rightarrow V$ (resp. the inclusion $V \rightarrow T V$ ). The construction carries over to augmented graded quivers and augmented differential graded quivers.

Denote by aQu the category of augmented $k$-quivers and by aCo that of cocomplete augmented $k$-cocategories. The forgetful functor $F: \mathrm{aCo} \rightarrow \mathrm{aQu}$ is monoidal.

Lemma 5.2. The forgetful functor admits the right adjoint $V \mapsto T V$. More precisely, for each augmented cocategory $C$ and each augmented $k$-quiver $V$, the map

$$
\operatorname{Hom}_{\mathrm{aco}}(C, T V) \rightarrow \operatorname{Hom}_{\mathrm{aQu}}(F C, V), f \mapsto p r_{1} \circ f
$$

is bijective. Its inverse takes a morphism $g$ to the unique morphism of augmented cocategories $G: C \rightarrow T V$ whose reduction sends $c \in \bar{C}$ to

$$
\sum_{n=1}^{\infty} g^{\otimes n} \Delta^{(n)}(c)
$$

The analogous assertions hold in the graded and in the differential graded setup. 
THEOREM 5.3. The monoidal category of cocomplete augmented cocategories admits an internal Hom-functor $\underline{\mathrm{Hom}}_{c}$. Moreover, if $C$ is a cocomplete augmented cocategory, then $\underline{\mathrm{Hom}}_{c}(C, T V)=T \underline{\mathrm{Hom}}_{q}(F C, V)$ for each augmented dg k-quiver $V$. The analogous assertions hold in the graded and in the differential graded setting.

Proof. We have to show that the functor ? $\otimes C$ admits a right adjoint, i.e. that the functor $\operatorname{Hom}\left(? \otimes C, C^{\prime}\right)$ is representable for each $C^{\prime}$. If $C^{\prime}=T V$, then

$$
\begin{aligned}
\operatorname{Hom}\left(? \otimes C, C^{\prime}\right) & =\operatorname{Hom}(? \otimes C, T V)=\operatorname{Hom}(F ? \otimes F C, V) \\
& =\operatorname{Hom}\left(F ?, \underline{\operatorname{Hom}}_{q}(F C, V)\right)=\operatorname{Hom}\left(?, T \underline{\operatorname{Hom}}_{q}(F C, V)\right) .
\end{aligned}
$$

If $C^{\prime}$ is arbitrary, we can write it as the equalizer of a pair of morphisms between cofree objects:

$$
T V_{1} \rightarrow T V_{2}
$$

Then we obtain the representing object $\underline{\operatorname{Hom}}_{c}\left(C, C^{\prime}\right)$ as the equalizer of the pair

$$
\underline{H o m}_{c}\left(?, T V_{1}\right) \stackrel{\rightarrow H o m}{H}_{c}\left(?, T V_{2}\right) .
$$

5.3. Explicit description of $\underline{\mathrm{Hom}}_{c}(C, T V)$ and the action morphism. Let $C$ be a cocomplete augmented cocategory and $V$ an augmented $k$-quiver. For $D=T V$, we will describe $\underline{\operatorname{Hom}}_{c}(C, D)$ and the adjunction morphism (=action morphism)

$$
\Phi: \underline{\operatorname{Hom}}_{c}(C, D) \otimes C \rightarrow D .
$$

These generalize V. Lyubashenko's constructions [27. The objects of

$$
\underline{H o m}_{c}(C, T V)=T \underline{H o m}_{c}(F C, V) \text {. }
$$

are the same as those of $\underline{H o m}_{q}(F C, V)$. Thus they are in bijection with the morphisms of augmented $k$-quivers $f$ from $C$ to $V$. The morphism $\eta$ of $T \underline{H o m}_{q}(F C, V)$ is obtained by composing that of $\underline{\operatorname{Hom}}_{q}(F C, V)$ with the canonical inclusion and dually, the morphism $\varepsilon$ by composing that of $\underline{\operatorname{Hom}}_{q}(F C, V)$ with the projection. If $f$ and $g$ are two objects, a morphism from $f$ to $g$ in $T \underline{H o m}_{q}(F C, V)$ is given by a tensor product of $n \geq 0$ composable morphisms of $\underline{H o m}_{q}(F C, V)$. For $n=0$, this means that $f=g$ and that the morphism is a multiple of $\eta_{f, f}(1)=f$; for $n \geq 1$, the morphism is a tensor

$$
\left(\varphi_{n}, \ldots, \varphi_{1}\right)=\varphi_{n} \otimes \cdots \otimes \varphi_{1}
$$

of morphisms $\varphi_{i}$ from $f_{i-1}$ to $f_{i}$ in the reduction $\overline{\operatorname{Hom}_{q}(F C, V)}$, where $f_{0}=f$ and $f_{n}=g$. Thus, $\varphi_{i}$ belongs to

$$
\prod_{x, y \in \operatorname{obj}(C)} \operatorname{Hom}_{k}\left(C(x, y), V\left(f_{i-1}(x), f_{i}(y)\right)\right.
$$

and the composition

$$
\varepsilon_{f_{i-1}(x), f_{i}(y)} \circ\left(\varphi_{i}\right)_{x, y}
$$

vanishes for all $x, y$. The image under $\Delta$ of such a morphism $\left(\varphi_{n}, \ldots, \varphi_{1}\right)$ is

$$
\sum_{i=0}^{n}\left(\varphi_{n}, \ldots, \varphi_{i+1}\right) \otimes\left(\varphi_{i}, \ldots, \varphi_{1}\right) .
$$

Here, the terms for $i=0$ and $i=n$ are respectively

$$
\left(\varphi_{n}, \ldots, \varphi_{1}\right) \otimes f \text { and } g \otimes\left(\varphi_{n}, \ldots, \varphi_{1}\right),
$$


by definition of the morphism $\eta$ for $\underline{\operatorname{Hom}}_{q}(F C, V)$. When we apply an iterate $\Delta^{(N)}$ of $\Delta$ to $\left(\varphi_{n}, \ldots, \varphi_{1}\right)$, we obtain sums of tensor products of $N$ factors each of which may be reduced to some $f_{i}$. For example, we get, among others, the term

$$
f_{7} \otimes\left(\varphi_{7}\right) \otimes f_{6} \otimes\left(\varphi_{6}, \varphi_{5}\right) \otimes f_{4} \otimes f_{4} \otimes\left(\varphi_{4}, \varphi_{3}, \varphi_{2}\right) \otimes f_{1} \otimes\left(\varphi_{1}\right)
$$

for $n=7$ and $N=9$.

The adjunction morphism $\Phi: \underline{H o m}_{c}(C, T V) \otimes C \rightarrow T V$ is the unique morphism of cocomplete augmented cocategories whose image under the forgetful functor makes the square

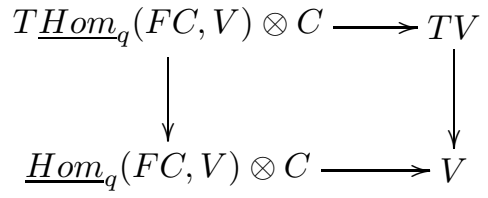

commutative in the category of augmented $k$-quivers. Thus, the morphism $\Phi$ takes an object $(f, x)$ of the tensor product $T \underline{\operatorname{Hom}}_{q}(F C, V) \otimes C$ to $f(x)$ and a morphism $\left(\varphi_{n}, \ldots, \varphi_{1}\right) \otimes c$ to the image in $\bigoplus_{N \geq n} \bar{V}^{\otimes N}$ of the sum of the

$$
\left(f_{n}^{\otimes r_{n}} \otimes \varphi_{n} \otimes f_{n-1}^{\otimes r_{n-1}} \otimes \cdots \otimes f_{1}^{\otimes r_{1}} \otimes \varphi_{1} \otimes f_{0}^{\otimes r_{0}}\right) \circ \Delta^{(N)}(c)
$$

where $N=n+\sum r_{i}$. Note that this sum has indeed only finitely many non zero terms: Since $C$ is cocomplete, $\Delta^{(M)}(c)$ vanishes in $\bar{C}^{\otimes M}$ for some $M \gg$ 0 . Therefore, $\Delta^{(N)}(c)$ vanishes in $C^{\otimes p} \otimes \bar{C}^{\otimes M} \otimes C^{\otimes q}$ for all $N=p+M+q$, $p, q \geq 0$. Now for large enough $N$, each term of the image of $\left(\varphi_{n}, \ldots, \varphi_{1}\right)$ under $\Delta^{(N)}$ will contain a chain of $M$ consecutive $f_{i}$ 's and therefore induce a map from $C^{\otimes p} \otimes \bar{C}^{\otimes M} \otimes C^{\otimes q}$ to $V^{\otimes p} \otimes \bar{V}^{\otimes M} \otimes V^{\otimes q}$. Hence the image of

$$
\Delta^{(N)}\left(\varphi_{n}, \ldots, \varphi_{1}\right) \circ \Delta^{(N)}(c)
$$

in $\bar{V}^{\otimes N}$ vanishes.

Note that if $C$ is the unit object $E$, the action morphism $\underline{\operatorname{Hom}}_{c}(E, D) \otimes E \rightarrow D$ is the identity. More generally, if $C$ is non empty, then $E$ is a retract of $C$ and the action morphism $\underline{\mathrm{Hom}}_{c}(C, D) \otimes C \rightarrow D$ admits a section. Dually, the coaction morphism $D \rightarrow \underline{\operatorname{Hom}}_{c}(C, D \otimes C)$ admits a retraction in this case.

5.4. The composition morphism. Let $C$ be a cocomplete augmented cocategory and $V, W$ augmented $k$-quivers. Since the functor ? $\otimes C$ is left adjoint to $\underline{H o m}_{c}(?, C)$, there is a unique morphism, the composition morphism,

$$
\zeta: \underline{H o m}_{c}(T V, T W) \otimes \underline{H o m}_{c}(C, T V) \longrightarrow \underline{H o m}_{c}(C, T W)
$$

which yields a commutative square

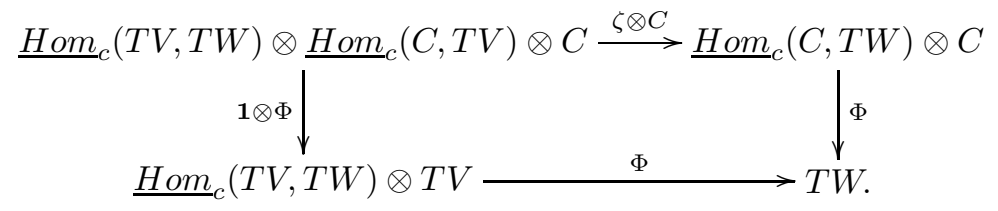


The composition morphism is constructed as the unique morphism such that the following square commutes in the category of augmented $k$-quivers

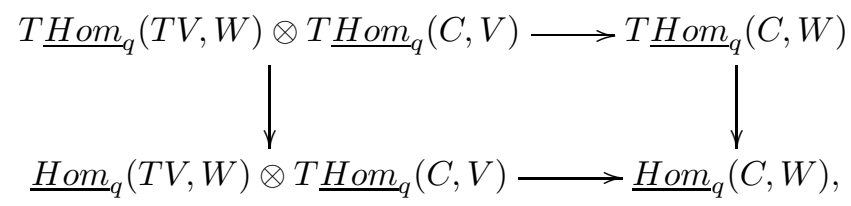

where the bottom arrow is described as follows: a pair $\left(f, f^{\prime}\right)$ of morphisms is sent to the composition $f \circ f^{\prime}$. A morphism $\varphi^{\prime} \otimes\left(\varphi_{n}, \ldots, \varphi_{1}\right)$ is sent to the morphism

$$
c \mapsto \sum \varphi^{\prime} \circ\left(f_{n}^{\otimes r_{n}} \otimes \varphi_{n} \otimes \cdots \otimes f_{i}^{\otimes r_{i}} \otimes \varphi_{i} \otimes f_{i-1}^{\otimes r_{i-1}} \otimes \cdots \otimes \varphi_{1} \otimes f_{0}^{\otimes r_{0}}\right) \circ \Delta^{(N)}(c)
$$

where $N$ is the number of arguments of $\varphi^{\prime}$ and $n+\sum r_{i}=N$.

5.5. The comparison morphism. Let $C$ be a cocomplete augmented cocategory and $W$ an augmented $k$-quiver. The comparison morphism is the composition

$$
F \underline{H o m}_{c}(C, T W) \rightarrow \underline{H o m}_{q}\left(F C, F \underline{H o m}_{c}(C, T W) \otimes F C\right) \rightarrow \underline{H o m}_{q}(F C, F T W)
$$

of the coaction morphism for ? $\otimes F C$ with the action morphism for ? $\otimes C$. This morphism induces the natural injection in the sets of objects. It takes a morphism $\left(\varphi_{n}, \ldots, \varphi_{1}\right), n \geq 1$, of $T \underline{\operatorname{Hom}}_{q}(C, W)$ to the morphism of $\underline{\operatorname{Hom}}_{q}(F C, F T W)$ which sends $c \in C(x, y)$ to the image in $\bigoplus_{N \geq n} \bar{W}^{\otimes N}$ of the sum of the

$$
\left(f_{n}^{\otimes r_{n}} \otimes \varphi_{n} \otimes f_{n-1}^{\otimes r_{n-1}} \otimes \cdots \otimes f_{1}^{\otimes r_{1}} \otimes \varphi_{1} \otimes f_{0}^{\otimes r_{0}}\right) \circ \Delta^{(N)}(c)
$$

where $N=n+\sum r_{i}$. We have seen above that this sum has only finitely many non zero terms. If the map $C \rightarrow \bar{C}^{\otimes n}$ induced by the iterated comultiplication is surjective for all $n \geq 2$, then the comparison morphism is clearly injective on the sets of morphisms. This happens for example if $C=T V$ for some augmented $k$-quiver $V$. The comparison morphism is compatible with compositions.

5.6. The differential graded case. Let adgCo denote the category of cocomplete augmented dg coalgebras and agCo the category of cocomplete augmented graded coalgebras. The functor

$$
\text { For : adgCo } \rightarrow \text { agCo }
$$

which forgets the differentials is monoidal. Thus, if $A, B$ are objects of adgCo, the adjunction morphism

$$
\underline{\operatorname{Hom}}_{q}(A, B) \otimes A \rightarrow B
$$

yields a morphism

$$
\text { For } \underline{\operatorname{Hom}}_{q}(A, B) \otimes \text { For } A \rightarrow \text { For } B
$$

and hence a morphism

$$
\text { For } \underline{\operatorname{Hom}}_{q}(A, B) \rightarrow \underline{\operatorname{Hom}}_{q}(\text { For } A \text {, For } B) \text {. }
$$

LEMMA 5.4. The above morphism induces isomorphisms in the morphism spaces, i. e. For $\underline{\operatorname{Hom}}_{q}(A, B)$ is the full subcocategory of $\underline{\operatorname{Hom}}_{q}($ For $A$, For $B)$ whose objects are the morphisms of agCo which commute with the differential. 
Proof. It suffices to prove this when $B$ is cofree on some augmented differential graded $k$-quiver $V$. Then we have

$$
\text { For } \underline{\operatorname{Hom}}_{q}(A, T V)=\text { For } T \underline{\operatorname{Hom}}_{q}(A, V)=T \text { For } \underline{\operatorname{Hom}}_{q}(A, V)
$$

where $\underline{\operatorname{Hom}}_{q}(A, V)$ is the internal $\underline{\operatorname{Hom}}_{q}$-object of the category of augmented differential graded $k$-quivers and we have also denoted by For the forgetful functor from $\operatorname{dg} k$-quivers to graded $k$-quivers. Now it is clear that we have a full morphism of graded quivers

$$
\text { For } \underline{\operatorname{Hom}}_{q}(A, V) \rightarrow \underline{\operatorname{Hom}}_{q}(\text { For } A \text {, For } V)
$$

and this implies the assertion.

5.7. $A_{\infty}$-functor categories. For an augmented graded $k$-quiver $V$, denote by $S V$ the augmented graded $k$-quiver with the same objects whose reduction has the shifted $k$-modules $S \bar{V}(x, y)$ as morphism spaces. An augmented $A_{\infty}$-category is an augmented graded $k$-quiver $A$ whose bar construction $B A=T S A$ is endowed with a graded coalgebra differential so that it becomes a cocomplete augmented dg cocategory. Equivalently, the reduction $\bar{A}$ is endowed with a structure of $A_{\infty}$ category without units in the sense of Kontsevich-Soibelman 24 4.1]. For example, if $A=k \oplus \bar{A}$ is an ordinary augmented associative algebra, then we view it as an $A_{\infty}$-category with one object by endowing

$$
B A=T S A=k \oplus \bigoplus_{n \geq 1}(S \bar{A})^{\otimes n}
$$

with the unique coalgebra differential such that $d(x \otimes y)=x y$ for $x, y \in \bar{A}$. Then the underlying complex of $B A$ is

$$
k \leftarrow \bar{A} \leftarrow \bar{A} \otimes \bar{A} \leftarrow \cdots
$$

and $H_{*}(B A)=\operatorname{Tor}_{*}^{A}(k, k)$.

Let $A, A^{\prime}$ be two augmented $A_{\infty}$-categories. By theorem [5.3] the cocategory $\underline{\operatorname{Hom}}_{c}\left(B A, B A^{\prime}\right)$ is (canonically) cofree. We define the $A_{\infty}$-functor category $\operatorname{Fun}_{\infty}\left(A, A^{\prime}\right)$ by

$$
\underline{\operatorname{Hom}}_{c}\left(B A, B A^{\prime}\right)=B\left(\operatorname{Fun}_{\infty}\left(A, A^{\prime}\right)\right) .
$$

It is an augmented $A_{\infty}$-category. By definition, its objects are the $A_{\infty}$-functors from $A$ to $A^{\prime}$. According to theorem 5.3 and the above lemma, the underlying graded category of $\underline{\operatorname{Hom}}_{c}\left(B A, B A^{\prime}\right)$ is a full subcategory of

$$
\underline{H o m}_{c}\left(B A, T S A^{\prime}\right)=T \underline{H o m}_{q}\left(B A, S A^{\prime}\right) \text {, }
$$

Thus, the underlying graded quiver of $S \operatorname{Fun}_{\infty}\left(A, A^{\prime}\right)$ is a full subquiver of

$$
\underline{\operatorname{Hom}}_{q}\left(B A, S A^{\prime}\right) \text {. }
$$

Suppose that $A$ and $A^{\prime}$ are ordinary augmented algebras. Then the objects of Fun $_{\infty}\left(A, A^{\prime}\right)$ identify with the morphisms of augmented dg coalgebras $B A \rightarrow B A^{\prime}$. For any two objects $f, g$, the morphism space

$$
S \operatorname{Fun}_{\infty}\left(A, A^{\prime}\right)(f, g)
$$

is

for $f \neq g$ and

$$
\operatorname{Hom}\left(k, S \overline{A^{\prime}}\right) \oplus \operatorname{Hom}\left(\overline{B A}, S \overline{A^{\prime}}\right)
$$

$$
k \oplus \operatorname{Hom}\left(k, S \overline{A^{\prime}}\right) \oplus \operatorname{Hom}\left(\overline{B A}, S \overline{A^{\prime}}\right)
$$


for $f=g$. If $f \neq g$, then the morphisms $f \rightarrow g$ are in canonical bijection with the space of $(f, g)$-coderivations $D: B A \rightarrow B A^{\prime}$, i. e. the homogeneous linear maps satisfying

$$
\Delta \circ D=(f \otimes D+D \otimes g) \circ \Delta .
$$

Note that $\operatorname{Fun}_{\infty}\left(A, A^{\prime}\right)$ has infinitely many objects, in general, even though $A$ and $A^{\prime}$ only have one. If $A=A^{\prime}$ and $f=g$ is the identity functor then

$$
\operatorname{Fun}_{\infty}(A, A)(\mathbf{1}, \mathbf{1}) / k
$$

is the reduced Hochschild complex

$$
\bar{A} \rightarrow \operatorname{Hom}(\bar{A}, \bar{A}) \rightarrow \operatorname{Hom}(\bar{A} \otimes \bar{A}, \bar{A}) \rightarrow \ldots \rightarrow \operatorname{Hom}\left(\bar{A}^{\otimes p}, \bar{A}\right) \rightarrow \ldots
$$

where $\bar{A}$ appears in degree -1 .

If $A, A^{\prime}, A^{\prime \prime}$ are three augmented $A_{\infty}$-categories, the composition morphism

$$
\underline{H o m}_{c}\left(B A^{\prime}, B A^{\prime \prime}\right) \otimes \underline{H o m}_{c}\left(B A, B A^{\prime}\right) \rightarrow \underline{H o m}_{c}\left(B A, B A^{\prime \prime}\right)
$$

yields a morphism

$$
B \operatorname{Fun}_{\infty}\left(A^{\prime}, A^{\prime \prime}\right) \otimes B \operatorname{Fun}_{\infty}\left(A, A^{\prime}\right) \rightarrow B \operatorname{Fun}_{\infty}\left(A, A^{\prime \prime}\right),
$$

which we view as an ' $A_{\infty}$-bifunctor'

$$
\operatorname{Fun}_{\infty}\left(A^{\prime}, A^{\prime \prime}\right) \times \operatorname{Fun}_{\infty}\left(A, A^{\prime}\right) \rightarrow \operatorname{Fun}_{\infty}\left(A, A^{\prime \prime}\right) .
$$

Let us show how this bifunctor yields the bialgebra structure on the bar construction of the Hochschild complex.

We need the following remark on cocategories: Let $\mathcal{C}$ be an augmented $k$ cocategory and $x$ and object of $\mathcal{C}$. Then the endomorphism space $\mathcal{C}(x, x)$ is not, in general, a coalgebra in a natural way, because the comultiplication

$$
\Delta: \mathcal{C}(x, x) \rightarrow \bigoplus_{y} \mathcal{C}(y, x) \otimes \mathcal{C}(x, y)
$$

does not take values in $\mathcal{C}(x, x) \otimes \mathcal{C}(x, x)$. However, if we define $\operatorname{End}(x)$ as the set of all $c \in \mathcal{C}(x, x)$ such that $\Delta^{(N)}(c)$ lies in $\mathcal{C}(x, x)^{\otimes N}$ for all $N \geq 2$, then $\operatorname{End}(x)$ becomes an augmented coalgebra, which we call the endomorphism coalgebra of $x$.

Now let $A$ be an ordinary augmented algebra and consider the cocategory

$$
\mathcal{C}=\underline{\operatorname{Hom}}_{c}(B A, B A)=B \operatorname{Fun}_{\infty}(A, A) .
$$

Then the endomorphism coalgebra of the identity functor $\operatorname{End}(\mathbf{1})$ is the bar construction $B(C(A, A))$, on the reduced Hochschild complex $C(A, A)$, i. e.

$$
\bar{A} \rightarrow \operatorname{Hom}(\bar{A}, \bar{A}) \rightarrow \operatorname{Hom}(\bar{A} \otimes \bar{A}, \bar{A}) \rightarrow \ldots \rightarrow \operatorname{Hom}\left(\bar{A}^{\otimes p}, \bar{A}\right) \rightarrow \ldots
$$

with $\bar{A}$ appearing in degree 0 . The composition bifunctor

$$
B \operatorname{Fun}_{\infty}(A, A) \otimes B \operatorname{Fun}_{\infty}(A, A) \rightarrow B \operatorname{Fun}_{\infty}(A, A)
$$

then yields an associative multiplication on the endomorphism coalgebra

$$
\operatorname{End}(\mathbf{1})=B(C(A, A))
$$

so that it becomes a bialgebra. Using the explicit description of the composition morphism in section 5.4 one checks that this bialgebra structure is given by the brace operations 11 17 [10, 11. 
5.8. Modules and bimodules. Let $\mathcal{C}(k)$ denote the dg category of complexes of vector spaces and $\mathcal{C}(k)^{+}$the augmented dg category whose reduction is $\mathcal{C}(k)$. Let $A$ be an augmented $A_{\infty}$-category. By definition, the category of (left) $A_{\infty}$-modules over $A$ is the category

$$
\operatorname{Fun}_{\infty}\left(A, \mathcal{C}(k)^{+}\right) .
$$

It is in fact a dg category (since the target category is a dg category). If $A^{\prime}$ is another augmented $A_{\infty}$-category, then we can consider

$$
\operatorname{Fun}_{\infty}\left(A^{\prime}, \operatorname{Fun}_{\infty}\left(A, \mathcal{C}(k)^{+}\right)\right) .
$$

From the canonical isomorphism

$$
\underline{H o m}_{c}\left(U, \underline{H o m}_{c}(V, W)\right) \stackrel{\sim}{\rightarrow} \underline{H o m}_{c}(U \otimes V, W)
$$

in the category of cocomplete augmented dg cocategories, we obtain

$$
\operatorname{Fun}_{\infty}\left(A^{\prime}, \operatorname{Fun}_{\infty}\left(A, \mathcal{C}(k)^{+}\right) \stackrel{\sim}{\rightarrow} \underline{\operatorname{Hom}}_{c}\left(B A^{\prime} \otimes B A, \mathcal{C}(k)^{+}\right),\right.
$$

and the last term is the category of $A_{\infty}$-bimodules. Equivalences of this type are quite unpleasant to prove directly, $c f$. for example Lemme 5.3.0.1 of [25].

\section{References}

[1] H. J. Baues, The double bar and cobar constructions, Compositio Math. 43 (1981), no. 3, 331-341.

[2] Alexander Beilinson, Victor Ginzburg, and Wolfgang Soergel, Koszul duality patterns in representation theory, J. Amer. Math. Soc. 9 (1996), no. 2, 473-527.

[3] David Benson, Henning Krause, and Stefan Schwede, Realizability of modules over Tate cohomology, Trans. Amer. Math. Soc. 356 (2004), no. 9, 3621-3668 (electronic).

[4] A. A. Beullinson, Coherent sheaves on $\mathbf{P}^{n}$ and problems in linear algebra, Funktsional. Anal. i Prilozhen. 12 (1978), no. 3, 68-69.

[5] A. Bondal and M. van den Bergh, Generators and representability of functors in commutative and noncommutative geometry, Mosc. Math. J. 3 (2003), no. 1, 1-36, 258.

[6] Joseph Chuang and Alastair King, The $A_{\infty}$-structure on the Yoneda algebra of a monomial algebra, preliminary notes.

[7] W. G. Dwyer and J. Spaliński, Homotopy theories and model categories, Handbook of algebraic topology, North-Holland, Amsterdam, 1995, pp. 73-126.

[8] Gunnar Floystad, Koszul duality and equivalences of categories, arXiv:math.RA/0012264

[9] Kenji Fukaya, Morse homotopy, $A^{\infty}$-category, and Floer homologies, Proceedings of GARC Workshop on Geometry and Topology '93 (Seoul, 1993) (Seoul), Lecture Notes Ser., vol. 18, Seoul Nat. Univ., 1993, pp. 1-102.

[10] Ezra Getzler, Cartan homotopy formulas and the Gauss-Manin connection in cyclic homology, Quantum deformations of algebras and their representations (Ramat-Gan, 1991/1992; Rehovot, 1991/1992), Israel Math. Conf. Proc., vol. 7, Bar-Ilan Univ., Ramat Gan, 1993, pp. 65-78.

[11] Ezra Getzler and J. D. S. Jones, Operads, homotopy algebra, and iterated integrals for double loop spaces, hep-th/9403055

[12] Ezra Getzler and John D. S. Jones, $A_{\infty}$-algebras and the cyclic bar complex, Illinois J. Math. 34 (1990), no. 2, 256-283.

[13] Vladimir Hinich, Homological algebra of homotopy algebras, Comm. Algebra 25 (1997), no. 10, 3291-3323.

[14] Mark Hovey, Model categories, Mathematical Surveys and Monographs, vol. 63, American Mathematical Society, Providence, RI, 1999.

[15] Johannes Huebschmann, The homotopy type of $F \Psi^{q}$. The complex and symplectic cases, Applications of algebraic $K$-theory to algebraic geometry and number theory, Part I, II (Boulder, Colo., 1983), Contemp. Math., vol. 55, Amer. Math. Soc., Providence, RI, 1986, pp. $487-518$.

[16] - The mod-p cohomology rings of metacyclic groups, J. Pure Appl. Algebra 60 (1989), no. $1,53-103$. 
[17] T. V. Kadeishvili, The structure of the $A(\infty)$-algebra, and the Hochschild and Harrison cohomologies, Trudy Tbiliss. Mat. Inst. Razmadze Akad. Nauk Gruzin. SSR 91 (1988), 1927.

[18] T. V. Kadeišvili, On the theory of homology of fiber spaces, Uspekhi Mat. Nauk 35 (1980), no. 3(213), 183-188, International Topology Conference (Moscow State Univ., Moscow, 1979).

[19] Bernhard Keller, Introduction to A-infinity algebras and modules, Homology Homotopy Appl. 3 (2001), no. 1, 1-35 (electronic).

[20] _ Addendum to: "Introduction to A-infinity algebras and modules" [Homology Homotopy Appl. 3 (2001), no. 1, 1-35; Homology Homotopy Appl. 4 (2002), no. 1, 25-28 (electronic).

[21] Maxim Kontsevich, Homological algebra of mirror symmetry, Proceedings of the International Congress of Mathematicians, Vol. 1, 2 (Zürich, 1994) (Basel), Birkhäuser, 1995, pp. 120-139.

[22] — Triangulated categories and geometry, Course at the École Normale Supérieure, Paris, Notes taken by J. Bellaïche, J.-F. Dat, I. Marin, G. Racinet and H. Randriambololona, 1998.

[23] Maxim Kontsevich and Yan Soibelman, Deformations of algebras over operads and the Deligne conjecture, Conférence Moshé Flato 1999, Vol. I (Dijon), Math. Phys. Stud., vol. 21, Kluwer Acad. Publ., Dordrecht, 2000, pp. 255-307.

[24] _ Homological mirror symmetry and torus fibrations, Symplectic geometry and mirror symmetry (Seoul, 2000), World Sci. Publishing, River Edge, NJ, 2001, pp. 203-263.

[25] Kenji Lefèvre-Hasegawa, Sur les $A_{\infty}$-catégories, Thèse de doctorat, Université Denis Diderot - Paris 7, November 2003, available at Bernhard Keller's homepage.

[26] D. M. Lu, J. H. Palmieri, Q. S. Wu, and J. J. Zhang, $A_{\infty}$-algebras for ring theorists, Proceedings of the International Conference on Algebra, vol. 11, 2004, pp. 91-128.

[27] Volodymyr Lyubashenko, Category of $A_{\infty}$-categories, Homology Homotopy Appl. 5 (2003), no. 1, 1-48 (electronic).

[28] Dag Madsen, Ph. d. thesis, NTNU, Trondheim, 2002.

[29] J. Peter May, Matric Massey products, J. Algebra 12 (1969), 533-568.

[30] Barry Mitchell, Rings with several objects, Advances in Math. 8 (1972), 1-161.

[31] Michael Penkava and Albert Schwarz, $A_{\infty}$ algebras and the cohomology of moduli spaces, Lie groups and Lie algebras: E. B. Dynkin's Seminar, Amer. Math. Soc. Transl. Ser. 2, vol. 169, Amer. Math. Soc., Providence, RI, 1995, pp. 91-107.

[32] Alain Prouté, Algèbres différentielles fortement homotopiquement associatives, Thèse d'État, Université Paris VII, 1984.

[33] Daniel G. Quillen, Homotopical algebra, Lecture Notes in Mathematics, No. 43, SpringerVerlag, Berlin, 1967.

[34] Jeremy Rickard, Morita theory for derived categories, J. London Math. Soc. 39 (1989), 436456.

[35] Raphaël Rouquier, Dimensions of triangulated categories, arXiv:math.CT/0310134

[36] Paul Seidel, Fukaya categories and deformations, Proceedings of the International Congress of Mathematicians, Vol. II (Beijing, 2002) (Beijing), Higher Ed. Press, 2002, pp. 351-360.

[37] V. A. Smirnov, Homology of fiber spaces, Uspekhi Mat. Nauk 35 (1980), no. 3(213), 227-230, International Topology Conference (Moscow State Univ., Moscow, 1979).

[38] James Dillon Stasheff, Homotopy associativity of $H$-spaces. I, II, Trans. Amer. Math. Soc. 108 (1963), 275-292; ibid. 108 (1963), 293-312.

[39] Jim Stasheff, Differential graded Lie algebras, quasi-Hopf algebras and higher homotopy algebras, Quantum groups (Leningrad, 1990), Lecture Notes in Math., vol. 1510, Springer, Berlin, 1992, pp. 120-137.

UFR de Mathématiques, Université Paris 7, Institut de Mathématiques, UMR 7586 Du CNRS, Case 7012, 2 Place Jussieu, 75251 Paris Cedex 05, France

E-mail address: keller@math.jussieu.fr 